\title{
Planetary system around the nearby M dwarf GJ 357 including a transiting, hot, Earth-sized planet optimal for atmospheric characterization ${ }^{\star}$
}

\author{
R. Luque ${ }^{1,2}$, E. Pallé ${ }^{1,2}$, D. Kossakowski ${ }^{3}$, S. Dreizler ${ }^{4}$, J. Kemmer ${ }^{5}$, N. Espinoza ${ }^{3}$, J. Burt ${ }^{6, \star \star}$, G. Anglada-Escudé ${ }^{7,8}$, \\ V. J. S. Béjar ${ }^{1,2}$, J. A. Caballero ${ }^{9}$, K. A. Collins ${ }^{10}$, K. I. Collins ${ }^{11}$, M. Cortés-Contreras ${ }^{9}$, E. Díez-Alonso ${ }^{12,13}$, \\ F. Feng ${ }^{14}$, A. Hatzes ${ }^{15}$, C. Hellier ${ }^{16}$, T. Henning ${ }^{3}$, S. V. Jeffers ${ }^{4}$, L. Kaltenegger ${ }^{17}$, M. Kürster ${ }^{3}$, J. Madden ${ }^{17}$, \\ K. Molaverdikhani ${ }^{3}$, D. Montes ${ }^{12}$, N. Narita ${ }^{1,18,19,20}$, G. Nowak ${ }^{1,2}$, A. Ofir ${ }^{21}$, M. Oshagh ${ }^{4}$, H. Parviainen ${ }^{1,2}$, \\ A. Quirrenbach ${ }^{5}$, S. Reffert ${ }^{5}$, A. Reiners ${ }^{4}$, C. Rodríguez-López ${ }^{8}$, M. Schlecker ${ }^{3}$, S. Stock ${ }^{5}$, T. Trifonov ${ }^{3}$, J. N. Winn ${ }^{22}$, \\ M. R. Zapatero Osorio ${ }^{23}$, M. Zechmeister ${ }^{4}$, P. J. Amado ${ }^{8}$, D. R. Anderson ${ }^{16}$, N. E. Batalha ${ }^{24}$, F. F. Bauer ${ }^{8}$, P. Bluhm ${ }^{5}$, \\ C. J. Burke ${ }^{6}$, R. P. Butler ${ }^{14}$, D. A. Caldwell ${ }^{25,26}$, G. Chen $^{27}$, J. D. Crane ${ }^{28}$, D. Dragomir ${ }^{6, \star \star \star}$, C. D. Dressing ${ }^{29}$, \\ S. Dynes ${ }^{6}$, J. M. Jenkins ${ }^{26}$, A. Kaminski ${ }^{5}$, H. Klahr ${ }^{3}$, T. Kotani ${ }^{18,20}$, M. Lafarga ${ }^{30,31}$, D. W. Latham ${ }^{10}$, P. Lewin ${ }^{32}$, \\ S. McDermott ${ }^{33}$, P. Montañés-Rodríguez ${ }^{1,2}$, J. C. Morales ${ }^{30,31}$, F. Murgas ${ }^{1,2}$, E. Nagel ${ }^{34}$, S. Pedraz ${ }^{35}$, I. Ribas ${ }^{30,31}$, \\ G. R. Ricker ${ }^{6}$, P. Rowden ${ }^{36}$, S. Seager ${ }^{6,37,38}$, S. A. Shectman $^{28}$, M. Tamura ${ }^{18,20,39}$, J. Teske $^{28, \star \star \star}$, J. D. Twicken ${ }^{25,26}$, \\ R. Vanderspeck ${ }^{6}$, S. X. Wang ${ }^{14}$, and B. Wohler ${ }^{25,26}$
}

(Affiliations can be found after the references)

Received 29 April 2019 / Accepted 27 June 2019

\begin{abstract}
We report the detection of a transiting Earth-size planet around GJ 357, a nearby M2.5 V star, using data from the Transiting Exoplanet Survey Satellite (TESS). GJ $357 \mathrm{~b}$ (TOI-562.01) is a transiting, hot, Earth-sized planet $\left(T_{\mathrm{eq}}=525 \pm 11 \mathrm{~K}\right)$ with a radius of $R_{\mathrm{b}}=1.217 \pm$ $0.084 R_{\oplus}$ and an orbital period of $P_{\mathrm{b}}=3.93$ d. Precise stellar radial velocities from CARMENES and PFS, as well as archival data from HIRES, UVES, and HARPS also display a 3.93-day periodicity, confirming the planetary nature and leading to a planetary mass of $M_{\mathrm{b}}=1.84 \pm 0.31 M_{\oplus}$. In addition to the radial velocity signal for GJ $357 \mathrm{~b}$, more periodicities are present in the data indicating the presence of two further planets in the system: GJ $357 \mathrm{c}$, with a minimum mass of $M_{\mathrm{c}}=3.40 \pm 0.46 M_{\oplus}$ in a $9.12 \mathrm{~d}$ orbit, and GJ $357 \mathrm{~d}$, with a minimum mass of $M_{\mathrm{d}}=6.1 \pm 1.0 M_{\oplus}$ in a $55.7 \mathrm{~d}$ orbit inside the habitable zone. The host is relatively inactive and exhibits a photometric rotation period of $P_{\text {rot }}=78 \pm 2 \mathrm{~d}$. GJ $357 \mathrm{~b}$ is to date the second closest transiting planet to the Sun, making it a prime target for further investigations such as transmission spectroscopy. Therefore, GJ $357 \mathrm{~b}$ represents one of the best terrestrial planets suitable for atmospheric characterization with the upcoming JWST and ground-based ELTs.
\end{abstract}

Key words. planetary systems - techniques: photometric - techniques: radial velocities - stars: individual: Gl 357 - stars: late-type

\section{Introduction}

To date nearly 200 exoplanets have been discovered orbiting approximately $100 \mathrm{M}$ dwarfs in the solar neighborhood (e.g., Bonfils et al. 2013; Rowe et al. 2014; Trifonov et al. 2018; Ribas et al. 2018). Some of these orbit near to or in the habitable zone (e.g., Udry et al. 2007; Anglada-Escudé et al. 2013, 2016; Tuomi \& Anglada-Escudé 2013; Dittmann et al. 2017; Reiners et al. 2018). However, only $11 \mathrm{M}$ dwarf planet systems have been detected with both the transit as well as the radial velocity (RV) method, which allows us to derive their density from their measured radius and mass, informing us about its bulk properties. When transit timing variation (TTV) mass measurements are included, TRAPPIST-1 (2MUCD 12171, Gillon et al. 2017) represents the 12th $\mathrm{M}$ dwarf planet system with mass and radius measurements.

\footnotetext{
* RV data are only available at the CDS via anonymous ftp to cdsarc.u-strasbg.fr $(130.79 .128 .5)$ or via http://cdsarc. u-strasbg.fr/viz-bin/qcat?J/A+A/628/A39

$\star \star$ Torres Fellow.

${ }^{\star \star \star}$ NASA Hubble Fellow.
}

Only six of the abovementioned eleven systems contain planets with masses below $10 M_{\oplus}$ : LHS $1140 \mathrm{~b}$ and $\mathrm{c}$ (GJ 3053, Dittmann et al. 2017; Ment et al. 2019), K2-3 b and c (PM J11293-0127, Almenara et al. 2015; Sinukoff et al. 2016), K2-18 b (PM J11302+0735, Cloutier et al. 2017; Sarkis et al. 2018), GJ 1214 b (LHS 3275 b, Harpsøe et al. 2013), GJ 1132 (Berta-Thompson et al. 2015; Bonfils et al. 2018), and b-g planets of TRAPPIST-1 (Gillon et al. 2016, 2017). However, only three planets with masses similar to Earth orbit M dwarfs of moderate brightness $(J=9.2-9.8 \mathrm{mag})$ : GJ $1132 \mathrm{~b}$ $\left(1.66 \pm 0.23 M_{\oplus}\right)$, LHS $1140 \mathrm{c}\left(1.81 \pm 0.39 M_{\oplus}\right)$, and K2-18 b $\left(2.1_{-13}^{+2.1} M_{\oplus}\right)$. Systems hosting small terrestrial exoplanets orbiting bright stars are ideal not only from the perspective of precise mass measurements with ground-based instruments, but also for further orbital (e.g., obliquity determination) and atmospheric characterization using current and future observatories (see, e.g., Batalha et al. 2018).

The Transiting Exoplanet Survey Satellite (TESS, Ricker et al. 2015) mission is an observatory that was launched to find small planets transiting small, bright stars. Indeed, since the start of scientific operations in July 2018, TESS has already 
uncovered over 600 new planet candidates, and is quickly increasing the sample of known Earths and super-Earths around small M-type stars (Vanderspek et al. 2019; Günther et al. 2019; Kostov et al. 2019). In this paper, we present the discovery of three small planets around a bright $\mathrm{M}$ dwarf, one of which, GJ 357 b, is an Earth-sized transiting exoplanet discovered using photometry from the TESS mission. To date, GJ $357 \mathrm{~b}$ is the second nearest $(d=9.44 \mathrm{pc})$ transiting planet to the Sun after HD 219134 b (Motalebi et al. 2015, $d=6.53$ pc), and the closest around an M dwarf. Besides, it is amenable to future detailed atmospheric characterization, opening the door to new studies for atmospheric characterization of Earth-like planet atmospheres (Pallé et al. 2009).

The paper is structured as follows. Section 2 presents the TESS photometry used for the discovery of GJ 357 b. Section 3 presents ground-based observations of the star including seeinglimited photometric monitoring, high-resolution imaging, and precise RVs. Section 4 presents a detailed analysis of the stellar properties of GJ 357. Section 5 presents an analysis of the available data in order to constrain the planetary properties of the system, including precise mass constraints on GJ $357 \mathrm{~b}$ along with a detection and characterization of two additional planets in the system, GJ 357 c and GJ 357 d. Section 6 presents a discussion of our results and, finally, Sect. 7 presents our conclusions.

\section{TESS photometry}

Planet GJ 357 (TIC 413248763) was observed by TESS in 2-min short-cadence integrations in Sector 8 (Camera \#2, CCD \#3) from February 2, 2019 until February 27, 2019 (see Fig. 1), and will not be observed again during the primary mission. At $\mathrm{BJD}=2458531.74$, an interruption in communications between the instrument and spacecraft occurred, resulting in an instrument turn-off until BJD $=2458535.00$. Together with the satellite repointing for data downlink between BJD $=2458529.06$ and $\mathrm{BJD}=2458530.44$, a gap of approximately $6 \mathrm{~d}$ is present in the photometry. In our analysis, the datapoints between $\mathrm{BJD}=2458530.44$ and $\mathrm{BJD}=2458531.74$ were masked out.

\subsection{Transit searches}

TESS objects of interest (TOIs) are announced regularly via the TESS data alerts public website ${ }^{1}$. TOI-562.01 was announced on April 13, 2019 and its corresponding light curve produced by the Science Processing Operations Center (SPOC; Jenkins et al. 2016) at the NASA Ames Research Center was uploaded to the Mikulski Archive for Space Telescopes (MAST) ${ }^{2}$ on April 17, 2019. SPOC provided for this target simple aperture photometry (SAP) and systematics-corrected photometry, a procedure consisting of an adaptation of the Kepler Presearch Data Conditioning algorithm (PDC, Smith et al. 2012; Stumpe et al. 2012, 2014) to TESS. The light curves generated by both methods are shown in Fig. 1. We use the latter one (PDC-corrected SAP, Fig. 1 bottom panel) for the remainder of this work.

A signal with a period of $3.93 \mathrm{~d}$ and a transit depth of $1164 \pm 66 \mathrm{ppm}$, corresponding to a planet radius of approximately $1.3 \pm 0.3 R_{\oplus}$ was detected in the TESS photometry. The Earth-sized planet candidate passed all the tests from the Alerts Data Validation Report ${ }^{3}$ (DVR; Twicken et al. 2018;

\footnotetext{
1 https://tess.mit.edu/alerts/

2 https://mast.stsci.edu

3 The complete DVR of TOI-562.01 can be downloaded from https://tev.mit.edu/vet/spoc-s08-b01/413248763/dl/ pdf/
}

Li et al. 2019), for example, even-odd transits comparison, eclipsing binary discrimination tests, ghost diagnostic tests to help rule out scattered light, or background eclipsing binaries, among others. The report indicates that the dimming events are associated with significant image motion, which is usually indicative of a background eclipsing binary. However, in this case, the reported information is meaningless because the star is saturated. On the other hand, the transit source is coincident with the core of the stellar point spread function (PSF), so the transit events happen on the target and not, for example, on a nearby bright star.

We also performed an independent analysis of the TESS light curve in order to confirm the DVR analysis and search for additional transit signals. An iterative approach was employed: in each iteration the same raw data were detrended and outliersrejected, a signal was identified and then modeled, and that model was temporarily divided-out during the detrending of the next iteration to produce a succession of improving models, until the $\chi^{2}$ converges. The raw photometry was detrended by fitting a truncated Fourier series, starting from the natural period of twice the data span, and all of its harmonics, down to some "protected" time span to make sure the filter does not modify the shape of the transit itself. We used a protected time span of $0.5 \mathrm{~d}$, and this series was iteratively fitted with $4 \sigma$ rejection. Finally, OptimalBLS (Ofir 2014) is used to identify the transit signal, which is then modeled using the Mandel \& Agol (2002) model and the differential evolution Markov chain Monte Carlo algorithm (ter Braak \& Vrugt 2008). The final model has $\chi_{v}^{2}=1.017$ and the resultant transit parameters are consistent with the TESS DVR. We also checked for odd-even differences between the transits, additional transit signals, and parabolic TTVs (Ofir et al. 2018) - all with null results.

\subsection{Limits on photometric contamination}

Given the large TESS pixel size of $21^{\prime \prime}$, it is essential to verify that no visually close-by targets are present that could affect the depth of the transit. There are two bright objects within one TESS pixel of GJ 357: (i) Gaia DR2 5664813824769090944 at $15.19^{\prime \prime}$ and $G_{R_{\mathrm{P}}}=15.57 \mathrm{mag}$; and (ii) Gaia DR2 5664814202726212224 at $18.31^{\prime \prime}$ and $G_{R_{\mathrm{P}}}=5.50 \mathrm{mag}$. However, they are much fainter than GJ 357 (TOI-562, Gaia DR2 $\left.5664814198431308288, G_{R_{\mathrm{P}}}=8.79 \mathrm{mag}\right)$ and their angular separations actually increased between the epochs of observation of Gaia (J2015.5) and TESS (J2019.1-J2019.2) due to the high proper motion of this star.

These two sources are by far the brightest ones apart from our target in the digitizations of red photographic plates taken in 1984 and 1996 with the UK Schmidt telescope. The Gaia $G_{R_{\mathrm{P}}}$-band (630-1050 nm) and the TESS band (600-1000 nm) are very much alike, allowing us to estimate the dilution factor for TESS using Eq. (2) in Espinoza et al. (2018) to be $D_{\text {TESS }}=0.996$, which is consistent with 1.00 , therefore compatible with no flux contamination.

\section{Ground-based observations}

\subsection{Transit follow-up}

We acquired ground-based time-series follow-up photometry of a full transit of TOI-562.01 on UTC April 26, 2019 from a Las Cumbres Observatory (LCO) $1.0 \mathrm{~m}$ telescope (Brown et al. 2013) at Cerro Tololo Inter-American Observatory (CTIO) as part of the TESS follow-up program (TFOP) SG1 Group. We used the TESS Transit Finder, which is a customized version of the Tapir software package (Jensen 2013), to schedule 


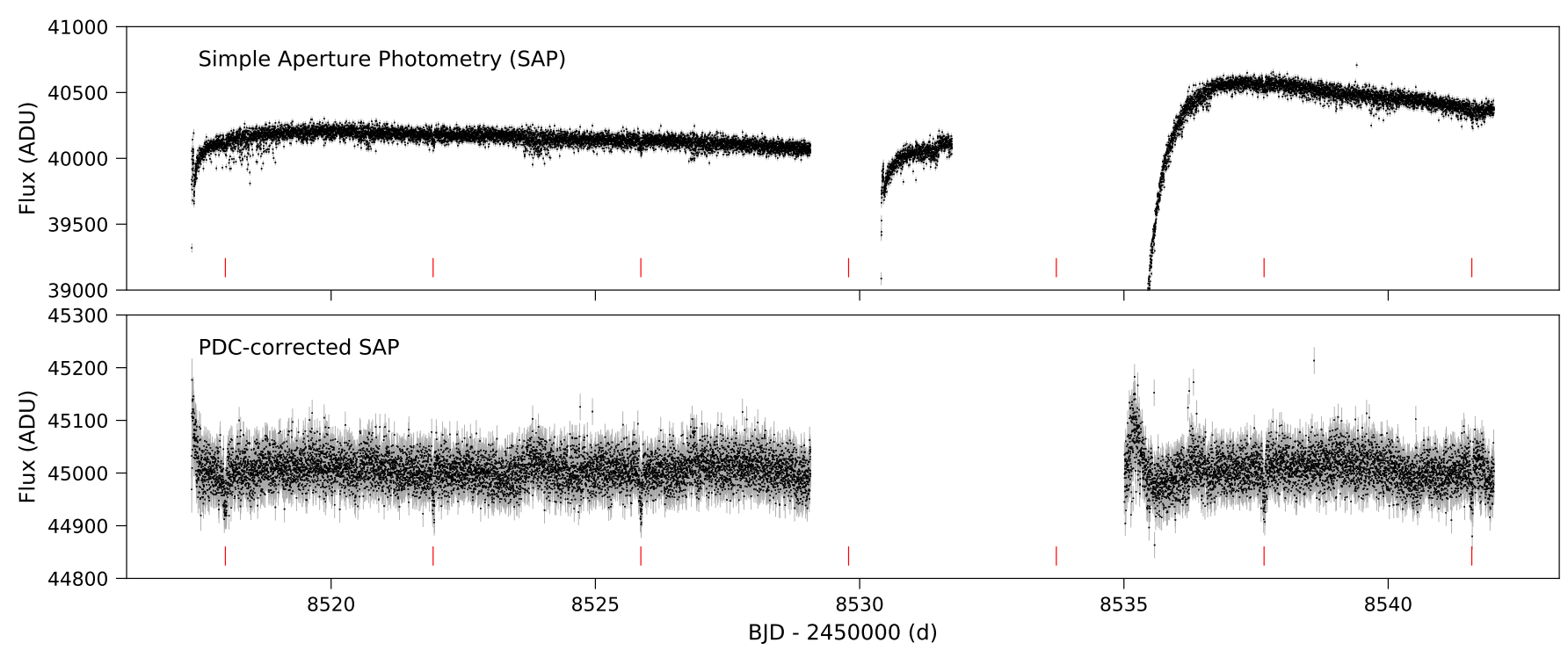

Fig. 1. TESS light curves of GJ 357 provided by SPOC. Top panel: simple aperture photometry. Bottom panel: PDC-corrected photometry. Transits of the planet candidate TOI-562.01 are marked in red.

photometric time-series follow-up observations. The $4096 \times$ 4096 LCO SINISTRO camera has an image scale of $0{ }^{\prime} 389$ pix $^{-1}$ resulting in a $26^{\prime} \times 26^{\prime}$ field of view. The $227 \mathrm{~min}$ observation in $z_{s}$ band used $30 \mathrm{~s}$ exposure times which, in combination with the $26 \mathrm{~s}$ readout time, resulted in 244 images. The images were calibrated by the standard LCO BANZAI pipeline and the photometric data were extracted using the AstroImageJ software package (Collins et al. 2017). The target star light curve shows a clear transit detection in a $7.78^{\prime \prime}$ radius aperture (see middle right panel of Fig. 5). The full width half maximum (FWHM) of the target and nearby stars is $\sim 4^{\prime \prime}$, so the follow-up aperture is only marginally contaminated by neighboring faint Gaia DR2 stars. The transit signal can be reliably detected with apertures that have a radius as small as $4.28^{\prime \prime}$, after which systematic effects start to dominate the light curve. We note that the detection of an $\sim 1200 \mathrm{ppm}$ transit with a $1 \mathrm{~m}$ ground-based telescope in a single transit is remarkable. A similar performance has been achieved only with the $1.2 \mathrm{~m}$ Euler-Swiss telescope combining two transits of HD 106315 c (Lendl et al. 2017), and highlights the importance of ground-based facilities to maintain and refine ephemeris of TESS planet candidates even in the Earth-sized regime.

\subsection{Seeing-limited photometric monitoring}

We made a compilation of photometric series obtained by longtime baseline, automated surveys exactly as in Díez Alonso et al. (2019). In particular we retrieved data from the following public surveys: All-Sky Automated Survey (ASAS; Pojmanski 2002), Northern Sky Variability Survey (NSVS; Woźniak et al. 2004), and All-Sky Automated Survey for Supernovae (ASAS-SN; Kochanek et al. 2017). The telescope location, instrument configurations, and photometric bands of each public survey were summarized by Díez Alonso et al. (2019). We did not find GJ 357 data in other public catalogs, such as The MEarth Project (Charbonneau et al. 2008), the Catalina surveys (Drake et al. 2014), or the Hungarian Automated Telescope Network (Bakos et al. 2004).

WASP-South, the southern station of the Wide Angle Search for Planets (Pollacco et al. 2006), is an array of eight cameras using 200-mm f/1.8 lenses backed by $2048 \times 2048$ CCDs, each camera covering $7.8^{\circ} \times 7.8^{\circ}$. It rasters a set of different pointings with a typical 10 -min cadence. WASP-South observed fields containing GJ 357 every year from 2007 to 2012, obtaining data over a span of typically $120 \mathrm{~d}$ each season, acquiring a total of 48000 photometric observations.

\subsection{High-resolution imaging}

FastCam. Although we discuss in Sect. 2.2 that there are no visually close companions that could affect the depth of the transit of GJ 357, we obtained high-resolution observations at different epochs to exclude the possibility of a physically-bound eclipsing binary that may produce the transits detected in the TESS light curve. First, we observed GJ 357 with the FastCam instrument (Oscoz et al. 2008) mounted on the $1.5 \mathrm{~m}$ Telescopio Carlos Sánchez at the Teide Observatory on January 14, 2013. These observations were part of our high-resolution imaging campaign of $\mathrm{M}$ dwarfs to characterize stellar multiplicity and select the most appropriate targets for the CARMENES survey (Cortés-Contreras et al. 2017). FastCam is a lucky imaging camera with a high readout speed, employing the subelectron noise L3CCD Andor $512 \times 512$ detector, which provides a pixel size of $0.0425^{\prime \prime}$ and a field of view of $21.2^{\prime \prime} \times 21.2^{\prime \prime}$. We obtained ten blocks of a thousand individual frames with $50 \mathrm{~ms}$ exposure time in the $I$ band. Data were bias subtracted, aligned, and combined using the brightest pixel as a reference as described in Labadie et al. (2010) and Jódar et al. (2013). We selected the best $10 \%$ of the frames to produce the final image and determined that there are no background contaminating sources with $\delta I<3$ mag down to $0.5^{\prime \prime}$ and with $\delta I<6 \mathrm{mag}$ down to $3.0^{\prime \prime}$ and up to $8.5^{\prime \prime}$ (given by the detector size).

IRD. We also observed GJ 357 with the InfraRed Doppler (IRD, Kotani et al. 2018) instrument on the Subaru $8.2 \mathrm{~m}$ telescope on April 18, 2019. IRD is a fibre-fed instrument through a fibre injection module behind an adaptive optics (AO) system (AO188, Hayano et al. 2010). A fibre injection module camera (FIMC) monitors images around targets to enable fibre injection of stellar light and guiding, and can take AO-corrected images of observing targets. The FIMC employs a CCD with pixel scale of $0.067^{\prime \prime}$ per pixel and observes in $970-1050 \mathrm{~nm}$. Figure 2 is the 


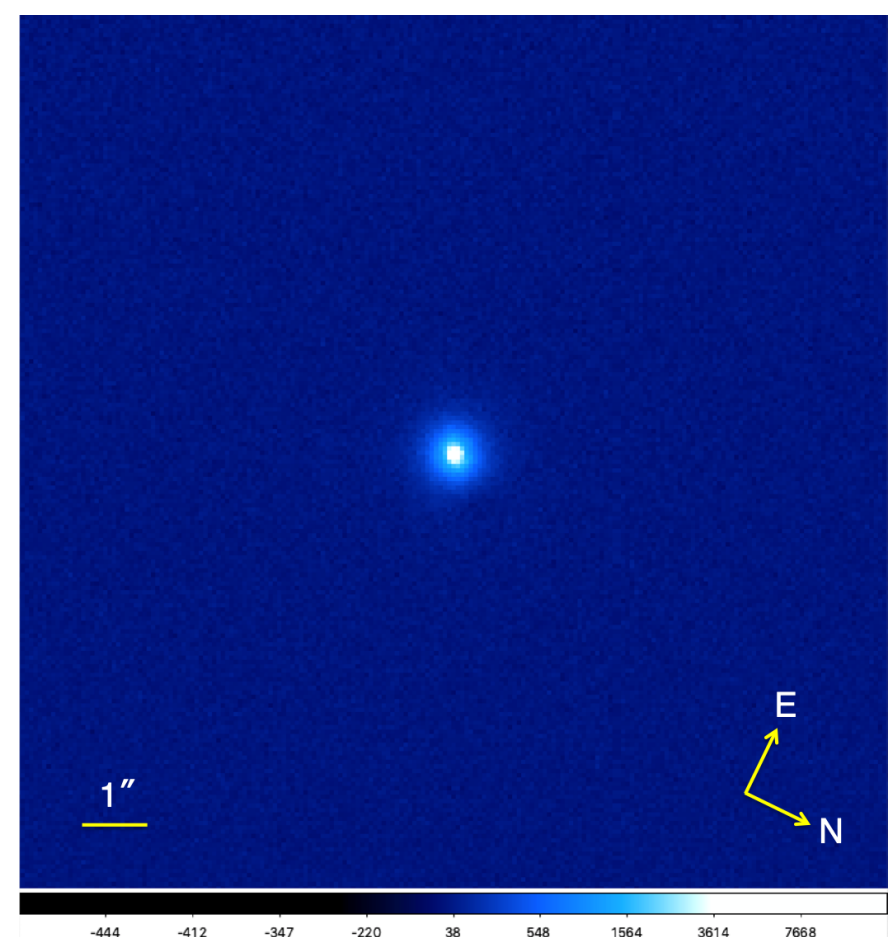

Fig. 2. Adaptive-optics-corrected image taken with the fiber injection module camera of IRD mounted on the Subaru $8.2 \mathrm{~m}$ telescope on April 18, 2019. The field of view is $13.4^{\prime \prime} \times 13.4^{\prime \prime}(200 \times 200$ pix $)$. Color coding is assigned in logarithmic scale. North is $116^{\circ}$ turned clockwise from the upper direction.

FIMC image of GJ 357. The image shows a $200 \times 200$ pixel region around GJ 357, revealing no nearby point source.

We note that GJ 357 is a high proper motion star with $0.139^{\prime \prime}$ per year in RA and -0.990" per year in Dec based on the Gaia Data Release 2 (DR2) data (Gaia Collaboration 2018). It means that the star was about $0.8^{\prime \prime}$ to the west and about $6^{\prime \prime}$ to the north at the time of the FastCam observation. The FastCam and IRD FIMC non-detection of any nearby companion excludes any background object at the original position of the FastCam observation. We exclude any false positive scenario and conclude there is no flux contamination from visually close-by targets in the GJ 357 transit data, and we fix the dilution factor for TESS to one in all of our model fits.

\subsection{Precise radial velocities}

\subsubsection{HIRES}

The high-resolution spectrograph HIRES (Vogt et al. 1994) mounted on the 10-m Keck-I telescope has been extensively used to search for exoplanets around bright dwarf stars using the RV technique (e.g., Vogt et al. 2000; Cumming et al. 2008). As part of this effort, Butler et al. (2017) published 64480 observations of a sample of 1699 stars collected with HIRES between 1996 and 2014. These data have been recently reanalyzed by Tal-Or et al. (2019) using a sample of RV-quiet stars (i.e., whose RV scatter is $<10 \mathrm{~m} \mathrm{~s}^{-1}$ ), who found small, but significant systematic effects in the RVs: a discontinuous jump caused by major modifications of the instrument in August 2004, a long-term drift, and a small intra-night drift. We use a total of 36 measurements for GJ 357 taken between January 26, 1998 and February 20, 2013. The RVs show a median internal uncertainty of $2.4 \mathrm{~m} \mathrm{~s}^{-1}$ and a rms of $4.0 \mathrm{~m} \mathrm{~s}^{-1}$ around the mean value.

\subsubsection{UVES}

Zechmeister et al. (2009) published 70 RV measurements of GJ 357 taken between November 15, 2000 and March 25, 2007 as part of the M dwarf planet search for terrestrial planets in the habitable zone with UVES at the ESO Very Large Telescope. RVs were obtained with the AUSTRAL code (Endl et al. 2000) and combined into nightly averages following Kürster et al. (2003). The 30 nightly binned RVs show a median internal uncertainty of $2.5 \mathrm{~m} \mathrm{~s}^{-1}$ and a rms of $5.3 \mathrm{~m} \mathrm{~s}^{-1}$ around the mean value.

\subsubsection{HARPS}

The High Accuracy Radial velocity Planet Searcher (HARPS, Mayor et al. 2003) is an ultra-precise Échelle spectrograph in the optical regime installed at the ESO $3.6 \mathrm{~m}$ telescope at La Silla Observatory in Chile, with a sub- $\mathrm{m} \mathrm{s}^{-1}$ precision. We retrieved 53 high-resolution spectra from the ESO public archive collected between December 13, 2003 and February 13, 2013. We extracted the FWHM and bisector span (BIS) of the crosscorrelation function from the FITS headers as computed by the DRS ESO HARPS pipeline (Lovis \& Pepe 2007), but to obtain the RVs we used SERVAL (Zechmeister et al. 2018), based on least-squares fitting with a high signal-to-noise (S/N) template created by co-adding all available spectra of the star. The RVs have a median internal uncertainty of $1 \mathrm{~m} \mathrm{~s}^{-1}$ and a rms of $3.3 \mathrm{~m} \mathrm{~s}^{-1}$ around the mean value.

\subsubsection{PFS}

The Planet Finder Spectrograph (Crane et al. 2010) is an iodine-cell, high-precision RV instrument mounted on the $6.5 \mathrm{~m}$ Magellan II telescope at Las Campanas Observatory in Chile. RVs are measured by placing a cell of gaseous $I_{2}$ in the converging beam of the telescope. This imprints the 5000-6200 $\AA$ region of incoming stellar spectra with a dense forest of $I_{2}$ lines that act as a wavelength calibrator, and provide a proxy for the PSF of the instrument. GJ 357 was observed a total of nine times as part of the long-term Magellan Planet Search Program between March 2016 and January 2019. After TESS' identification of transits in GJ 357, the star was then observed at higher precision during the April and May 2019 runs, which added an additional seven RVs to the dataset. The iodine data prior to February 2018 (PFSpre) were taken through a $0.5^{\prime \prime}$ slit resulting in $R \sim 80000$, and those after (PFSpost) were taken through a $0.3^{\prime \prime}$ slit, resulting in $R \sim 130000$. A different offset must be accounted for the RVs taken before and after this intervention. All PFS data are reduced with a custom IDL pipeline that flat fields, removes cosmic rays, and subtracts scattered light. Additional details about the iodine-cell RV extraction method can be found in Butler et al. (1996). The RVs have a median internal uncertainty of $1.3(0.7) \mathrm{m} \mathrm{s}^{-1}$ and an rms of $3.1(2.3) \mathrm{m} \mathrm{s}^{-1}$ around the mean value for PFSpre (PFSpost).

\subsubsection{CARMENES}

The star GJ 357 (Karmn J09360-216) is one of the 342 stars monitored in the CARMENES Guaranteed Time Observation program to search for exoplanets around M dwarfs, which began in January 2016 (Reiners et al. 2018). The CARMENES instrument is mounted at the $3.5 \mathrm{~m}$ telescope at the Calar Alto Observatory in Spain and has two channels: the visual (VIS) covers the spectral range $0.52-0.96 \mu \mathrm{m}$ and the near-infrared (NIR) covers the $0.96-1.71 \mu \mathrm{m}$ range (Quirrenbach et al. 2014, 2018). GJ 357 was observed ten times between December 13, 
2016 and March 16, 2019, and the VIS RVs - extracted with SERVAL and corrected for barycentric motion, secular acceleration, instrumental drift, and nightly zero-points (see Trifonov et al. 2018; Luque et al. 2018, for details) - show a median internal uncertainty of $1.3 \mathrm{~m} \mathrm{~s}^{-1}$ and a rms of $2.8 \mathrm{~m} \mathrm{~s}^{-1}$ around the mean value.

\section{Stellar properties}

\subsection{Stellar parameters}

The star GJ 357 (L 678-39, Karmn J09360-216, TIC 413248763) is a high proper motion star in the Hydra constellation classified as M2.5 V by Hawley et al. (1996). Located at a distance of $d \approx 9.4 \mathrm{pc}$ (Gaia Collaboration 2018), it is one of the brightest single $\mathrm{M}$ dwarfs in the sky, with an apparent magnitude in the $J$ band of 7.337 mag (Skrutskie et al. 2006) and no evidence for multiplicity, either at short or wide separations (CortésContreras et al. 2017). Accurate stellar parameters of GJ 357 were presented in Schweitzer et al. (2019), who determined radii, masses, and updated photospheric parameters for 293 bright $M$ dwarfs from the CARMENES survey using various methods. In summary, Schweitzer et al. (2019) derived the radii from Stefan-Boltzmann's law, effective temperatures from a spectral analysis using the latest grid of PHOENIX-ACES models, luminosities from integrating broadband photometry together with Gaia DR2 parallaxes, and masses from an updated mass-radius relation derived from eclipsing binaries.

According to this analysis, GJ 357 has an effective temperature of $3505 \pm 51 \mathrm{~K}$ and a mass of $0.342 \pm 0.011 M_{\odot}$. Furthermore, with the Gaia DR2 equatorial coordinates, proper motions, and parallax, and absolute RV measured from CARMENES spectra, we compute galactocentric space velocities $U V W$ as in Montes et al. (2001) and Cortés Contreras (2016) that kinematically put GJ 357 in the thin disk of the Galaxy. A summary of all stellar properties can be found in Table 1 .

\subsection{Activity and rotation period}

Using CARMENES data, Reiners et al. (2018) determined a Doppler broadening upper limit of $v \sin i<2 \mathrm{~km} \mathrm{~s}^{-1}$ for GJ 357 . This slow rotational velocity is consistent with its low level of magnetic activity. An analysis of the $\mathrm{H} \alpha$ activity in the CARMENES spectra shows that it is an inactive star and that the rotational variations in $\mathrm{H} \alpha$ and other spectral indicators are consistent with other inactive stars (Schöfer et al. 2019). GJ 357 has a $\log R_{\mathrm{HK}}^{\prime}$ value of -5.37 , and is one of the least active stars in the Boro Saikia et al. (2018) catalog of chromospheric activity of nearly 4500 stars, consistent with our kinematic analysis. In addition, this is in agreement with the upper limit set by Stelzer et al. (2013) in its X-ray flux $\left(\log F_{\mathrm{X}}<-13.09 \mathrm{~mW} \mathrm{~m}^{-2}\right)$ and the fact that Moutou et al. (2017) were not able to measure its magnetic field strength based on optical high-resolution spectra obtained with ESPaDOnS at the Canada-France-Hawai'i Telescope.

From spectroscopic determinations, the small value of $\log R_{\mathrm{HK}}^{\prime}$ indicates a long rotation period of between 70 and $120 \mathrm{~d}$ (Suárez Mascare no et al. 2015; Astudillo-Defru et al. 2017; Boro Saikia et al. 2018). Therefore, we searched the WASP data for rotational modulations, treating each season of data in a given camera as a separate dataset, using the methods presented in Maxted et al. (2011). The results are tabulated in Table 2. We find a significant 70-90 d periodicity across all seasons with more than 2000 datapoints. Since this timescale is not much shorter than the coverage in each year, the period error in each dataset
Table 1. Stellar parameters of GJ 357.

\begin{tabular}{|c|c|c|}
\hline$\underline{\text { Parameter }}$ & Value & Reference \\
\hline \multicolumn{3}{|c|}{ Name and identifiers } \\
\hline Name & L 678-39 & Luyten (1942) \\
\hline GJ & 357 & Gliese (1957) \\
\hline Karmn & J09360-216 & AF15 \\
\hline TOI & 562 & TESS Alerts \\
\hline TIC & 413248763 & Stassun et al. (2018) \\
\hline \multicolumn{3}{|c|}{ Coordinates and spectral type } \\
\hline$\alpha$ & 09:36:01.64 & Gaia DR2 \\
\hline$\delta$ & $-21: 39: 38.9$ & Gaia DR2 \\
\hline SpT & $\mathrm{M} 2.5 \mathrm{~V}$ & Hawley et al. (1996) \\
\hline \multicolumn{3}{|c|}{ Magnitudes } \\
\hline$B$ (mag) & $12.52 \pm 0.02$ & UCAC4 \\
\hline$V(\mathrm{mag})$ & $10.92 \pm 0.03$ & UCAC4 \\
\hline$g$ (mag) & $11.70 \pm 0.02$ & UCAC4 \\
\hline$G(\mathrm{mag})$ & $9.8804 \pm 0.0014$ & Gaia DR2 \\
\hline$r$ (mag) & $10.34 \pm 0.09$ & UCAC4 \\
\hline$i(\mathrm{mag})$ & $9.35 \pm 0.27$ & UCAC4 \\
\hline$J(\mathrm{mag})$ & $7.337 \pm 0.034$ & 2MASS \\
\hline$H$ (mag) & $6.740 \pm 0.033$ & 2MASS \\
\hline$K_{\mathrm{s}}(\mathrm{mag})$ & $6.475 \pm 0.017$ & 2MASS \\
\hline \multicolumn{3}{|c|}{ Parallax and kinematics } \\
\hline$\pi$ (mas) & $105.88 \pm 0.06$ & Gaia DR2 \\
\hline$d(\mathrm{pc})$ & $9.444 \pm 0.005$ & Gaia DR2 \\
\hline$\mu_{\alpha} \cos \delta\left(\operatorname{mas~yr}^{-1}\right)$ & $+138.694 \pm 0.100$ & Gaia DR2 \\
\hline$\mu_{\delta}\left(\operatorname{mas~yr}^{-1}\right)$ & $-990.311 \pm 0.083$ & Gaia DR2 \\
\hline$V_{r}\left(\mathrm{~km} \mathrm{~s}^{-1}\right)$ & $-34.70 \pm 0.50$ & This work \\
\hline$U\left(\mathrm{~km} \mathrm{~s}^{-1}\right)$ & $41.11 \pm 0.13$ & This work \\
\hline$V\left(\mathrm{~km} \mathrm{~s}^{-1}\right)$ & $11.37 \pm 0.45$ & This work \\
\hline$W\left(\mathrm{~km} \mathrm{~s}^{-1}\right)$ & $-37.25 \pm 0.19$ & This work \\
\hline \multicolumn{3}{|c|}{ Photospheric parameters } \\
\hline$T_{\text {eff }}(\mathrm{K})$ & $3505 \pm 51$ & Schweitzer et al. (2019) \\
\hline $\log g$ & $4.94 \pm 0.07$ & Schweitzer et al. (2019) \\
\hline$[\mathrm{Fe} / \mathrm{H}]$ & $-0.12 \pm 0.16$ & Schweitzer et al. (2019) \\
\hline$v \sin i_{\star}\left(\mathrm{km} \mathrm{s}^{-1}\right)$ & $<2.0$ & Reiners et al. (2018) \\
\hline \multicolumn{3}{|c|}{ Physical parameters } \\
\hline$M\left(M_{\odot}\right)$ & $0.342 \pm 0.011$ & Schweitzer et al. (2019) \\
\hline$R\left(R_{\odot}\right)$ & $0.337 \pm 0.015$ & Schweitzer et al. (2019) \\
\hline$L\left(10^{-4} L_{\odot}\right)$ & $159.1 \pm 3.6$ & Schweitzer et al. (2019) \\
\hline
\end{tabular}

References. AF15: Alonso-Floriano et al. (2015); Gaia DR2: Gaia Collaboration (2018); UCAC4: Zacharias et al. (2013); 2MASS: Skrutskie et al. (2006).

is $\sim 10 \mathrm{~d}$. The amplitude of the modulation ranges from 2 to $9 \mathrm{mmag}$, and the false-alarm probability is less than $10^{-4}$.

Then, we use a more sophisticated model to determine precisely the empirical rotational period of the star by fitting the full photometric dataset described in Sect. 3.2 (i.e., the ASAS, NSVS, ASAS-SN - with observations both in $g$ and $V$ bands and WASP datasets) with a quasi-periodic (QP) Gaussian process (GP). In particular, we use the GP kernel introduced in Foreman-Mackey et al. (2017) of the form

$k_{i, j}(\tau)=\frac{B}{2+C} e^{-\tau / L}\left[\cos \left(\frac{2 \pi \tau}{P_{\text {rot }}}\right)+(1+C)\right]$,

where $\tau=\left|t_{i}-t_{j}\right|$ is the time-lag, $B$ and $C$ define the amplitude of the GP, $L$ is a timescale for the amplitude-modulation of the GP, and $P_{\text {rot }}$ is the rotational period of the QP modulations. For the fit, we consider that each of the five datasets can have different values of $B$ and $C$ in order to account for the possibility 
Table 2. Rotation-modulation search of WASP-South data.

\begin{tabular}{lccccc}
\hline \hline Year (camera) & $N_{\text {pts }}$ & $\begin{array}{c}P \\
(\mathrm{~d})\end{array}$ & $\begin{array}{c}\text { Ampl. } \\
(\mathrm{mag})\end{array}$ & FAP & $\begin{array}{c}\mathrm{A}_{95}(a) \\
(\mathrm{mag})\end{array}$ \\
\hline $2007(226)$ & 7225 & 74 & 0.002 & 0.0099 & 0.0011 \\
$2008(226)$ & 8947 & 79 & 0.002 & 0.0083 & 0.0013 \\
$2009(228)$ & 5785 & 91 & 0.005 & 0.0000 & 0.0015 \\
$2010(227)$ & 2291 & 84 & 0.006 & 0.0001 & 0.0036 \\
$2010(228)$ & 4745 & 84 & 0.009 & 0.0000 & 0.0038 \\
$2011(222)$ & 5272 & 72 & 0.004 & 0.0000 & 0.0019 \\
$2012(222)$ & 5085 & 74 & 0.007 & 0.0000 & 0.0026 \\
$2012(227)$ & 2605 & 71 & 0.006 & 0.0000 & 0.0030 \\
\hline
\end{tabular}

Notes. ${ }^{(a)}$ Amplitude corresponding to a $95 \%$ probability of a false alarm.

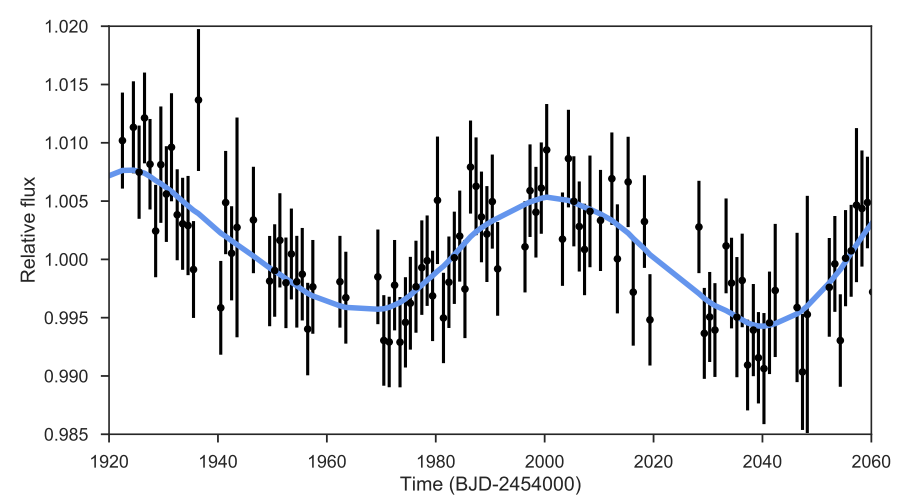

Fig. 3. Close-up of the GP fit to all the photometric datasets used to estimate the stellar rotation period of the star. Black points show the WASP data, where a QP modulation can be clearly seen. Our best-fit GP fit (blue) reveals a rotational period of $P_{\text {rot }}=77.8_{-2.0}^{+2.1} \mathrm{~d}$.

that different bands could have different GP amplitudes, while the timescale of the modulation as well as the rotational period is left as a common parameter between the datasets. In addition, we fit for a flux offset between the photometric datasets, as well as for extra jitter terms added in quadrature to the diagonal of the resulting covariance matrix implied by this QP GP. We consider wide priors for $B, C$ (log-uniform between $10^{-5} \mathrm{ppm}$ and $\left.10^{5} \mathrm{ppm}\right), L$ (log-uniform between $10^{-5}$ and $10^{5} \mathrm{~d}$ ), rotation period (uniform between 0 and $100 \mathrm{~d}$ ), flux offsets (Gaussian centered on 0 and standard deviation of $10^{5} \mathrm{ppm}$ ), and jitters ( $\log$-uniform between 1 and $10^{5} \mathrm{ppm}$ ). The fit is performed using juliet (Espinoza et al. 2018, see next section for a full description of the algorithm) and a close-up of the resulting fit is presented in Fig. 3 for illustration on how large the QP variations are in the WASP photometry, where the flux variability can be readily seen by eye.

The resulting rotational period from this analysis is of $P_{\text {rot }}=77.8_{-2.0}^{+2.1} \mathrm{~d}$, consistent with the expectation from the small value of $\log R_{\mathrm{HK}}^{\prime}$.

\section{Analysis and results}

\subsection{Period analysis of the RV data}

We performed a signal search in the RV data using generalized Lomb-Scargle (GLS) periodograms (Zechmeister \& Kürster 2009). Figure 4 presents a series of GLS periodograms of the residual RVs after subtracting an increasing number of periodic signals. For each panel, we computed the theoretical false alarm probability (FAP) as described in Zechmeister \& Kürster (2009), and show the 10,1 , and $0.1 \%$ levels. After subtracting a model that fits only the instrumental offsets $\mu_{\text {instr }}$ and jitters $\sigma_{\text {instr }}$ (Fig. 4a), we find that the periodogram is dominated by a periodic signal at $P \sim 56 \mathrm{~d}$ and its aliases around periods of one day due to the sampling of the data.

After fitting a sinusoid to this signal, a GLS periodogram of the residuals shows many signals with FAP $<1 \%$. One of those signals is at $3.93 \mathrm{~d}$, corresponding to the transiting planet detected in the TESS data. In this case, however, we want to know what is the probability that noise can produce a peak higher than what is seen exactly at the known frequency of the transiting planet, the spectral FAP. Following Zechmeister \& Kürster (2009), we use a bootstrapping randomization method over a narrow frequency range centered on the planet orbital frequency to determine it. The analysis yields spectral $\mathrm{FAP}=0.00075$. We thus estimate a FAP $\sim 0.08 \%$ for the 3.93 d signal.

The residuals after the modeling of the 56 and $3.93 \mathrm{~d}$ signals support a further periodicity of $P=9.1 \mathrm{~d}$ with a FAP $<0.1 \%$ (Fig. 4c). This signal is persistent throughout the complete analysis and therefore cannot be explained by any of the other two known sources. Including this periodicity in the model as an extra sinusoid (Fig. 4d), the GLS of the residuals reveals a single relevant periodicity at $87 \mathrm{~d}$. When including a fourth sinusoid in the analysis at $87 \mathrm{~d}$, different peaks with FAP $\approx 10 \%$ populate the $1 \mathrm{~d}$ region. We discuss in depth the nature of the four signals detected in the GLS in the next section, using more sophisticated models to fit the numerous periodicities in the RV dataset.

\subsection{Modeling results}

We used the recently published algorithm juliet (Espinoza et al. 2018) to model jointly the photometric and Doppler data. The algorithm is built on many publicly available tools for the modeling of transits (batman, Kreidberg 2015), RVs (radvel, Fulton et al. 2018), and GP (george, Ambikasaran et al. 2015; celerite, Foreman-Mackey et al. 2017). In order to compare different models, juliet efficiently computes the Bayesian model $\log$ evidence $(\ln Z)$ using either MultiNest (Feroz et al. 2009) via the PyMultiNest package (Buchner et al. 2014) or the dynesty package (Speagle 2019). Nested sampling algorithms sample directly from the given priors instead of starting off with an initial parameter vector around a likelihood maximum found via optimization techniques, as done in common sampling methods. The trade-off between its versatility and completeness in the parameter space search is the computation time. For this reason, our prior choices have been selected to be the ideal balance between being informed, yet wide enough to fully acquire the posterior distribution map. We consider a model to be moderately favored over another if the difference in its Bayesian log evidence is greater than two, and strongly favored if it is greater than five (Trotta 2008). If $\Delta \ln Z \lesssim 2$, then the models are indistinguishable so the simpler model with less degrees of freedom would be chosen.

\subsubsection{Photometry only}

In order to constrain the orbital period and time of transit center, we performed an analysis with juliet using only the TESS photometry. We chose the priors in the orbital parameters from the TESS DVR and our independent optimal BLS analysis. We adopted a few parametrization modifications when dealing with the transit photometry. Namely, we assigned a quadratic limb-darkening law for TESS, as shown to be appropriate for 


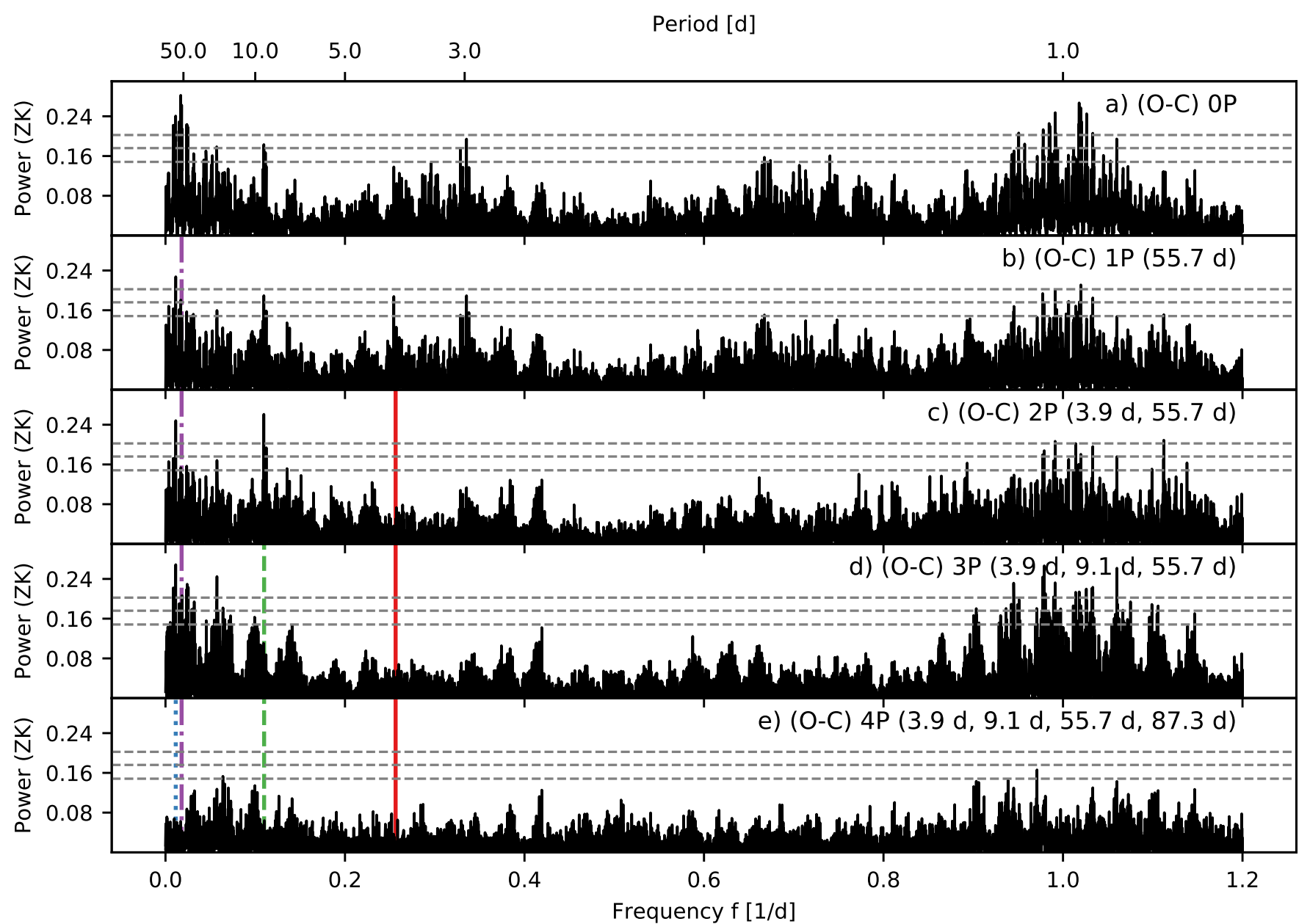

Fig. 4. Generalized Lomb-Scargle periodograms of the residual RVs after subtraction of different models. Panel a: no signal subtracted, only instrumental offsets and jitter fitted. Panel $b$ : periodogram of the RV residuals after the subtraction of one sinusoidal signal with $P=55.7 \mathrm{~d}$ (vertical purple dashed-dotted line). Panel $c$ : periodogram of the RV residuals after the simultaneous modeling of two signals with periods at $3.93 \mathrm{~d}$ (red solid line) and $55.7 \mathrm{~d}$. Panel $d$ : periodogram of the RV residuals after the simultaneous modeling of three periodic signals with $P=55.7 \mathrm{~d}, P=3.93 \mathrm{~d}$, and $P=9.1 \mathrm{~d}$ (green dashed line). Panel $e$ : periodogram of the RV residuals after the simultaneous modeling of four periodic signals with $P=55.7 \mathrm{~d}$, $P=3.93 \mathrm{~d}, P=9.1 \mathrm{~d}$, and $P \sim 87 \mathrm{~d}$ (blue dotted line). The gray dashed lines indicate from bottom to top the analytic 10,1 , and $0.1 \%$ FAP levels, respectively.

space-based missions (Espinoza \& Jordán 2015), which then was parametrized with the uniform sampling scheme $\left(q_{1}, q_{2}\right)$, introduced by Kipping (2013). Additionally, rather than fitting directly for the planet-to-star radius ratio $\left(p=R_{p} / R_{*}\right)$ and the impact parameter of the orbit $(b)$, we instead used the parametrization introduced in Espinoza (2018) and fit for the parameters $r_{1}$ and $r_{2}$ to guarantee full exploration of physically plausible values in the $(p, b)$ plane. Lastly, we applied the classical parametrization of $(e, \omega)$ into $\left(\mathcal{S}_{1}=\sqrt{e} \sin \omega\right.$, $\left.\mathcal{S}_{2}=\sqrt{e} \cos \omega\right)$, always ensuring that $e=\mathcal{S}_{1}^{2}+\mathcal{S}_{2}^{2} \leq 1$. We fixed the TESS dilution factor to one based on our analysis from Sects. 2.2 and 3.3, but accounted for any residual time-correlated noise in the light curve with an exponential GP kernel of the form $k_{i, j}=\sigma_{\mathrm{GP}, \mathrm{TESS}}^{2} \exp \left(-\left|t_{i}-t_{j}\right| / T_{\mathrm{GP}, \mathrm{TESS}}\right)$, where $T_{\mathrm{GP}, \mathrm{TESS}}$ is a characteristic timescale and $\sigma_{\mathrm{GP} \text {,TESS }}$ is the amplitude of this GP modulation. Furthermore, we added in quadrature a jitter term $\sigma_{\text {TESS }}$ to the TESS photometric uncertainties, which might be underestimated due to additional systematics in the spacebased photometry. The details of the priors and the description for each parameter are presented in Table A.1.

The results from the photometry-only analysis with juliet are completely consistent with those provided by the TESS DVR and our independent transit search, but with improved precision in the transit parameters after accounting for extra systematics with the jitter term and the GP. We also searched for an additional transiting planet in the system by modeling a two-planet fit where we use the same priors in Table A.1 for the first planet, and then allow the period and time of transit center to vary for the second. The transiting model for the hypothetical second planet is totally flat and we find no strong evidence $\left(\Delta \ln Z=\ln Z_{1 \mathrm{pl}}-\right.$ $\ln Z_{2 \mathrm{pl}}=5.16$ ) for any additional transiting planets in the light curve, in agreement with our findings in Sect. 2.1.

\subsubsection{RV only}

In Sect. 5.1, we have shown that several signals are present in the RV data. We tested several models using juliet on the RV dataset independently to understand the nature of those signals and their significance when doing a simultaneous multiplanetary fit. We discuss three sets of models, each exploring possible system architectures covering the four interesting periodicities from Sect. 5.1 of 3.93, 9.1, 55.7, and 87.3 d. The details regarding the priors and Bayesian log evidence of all the runs are listed in Table 3. We included an instrumental jitter term for each 
Table 3. Model comparison of RV-only fits with juliet.

\begin{tabular}{|c|c|c|c|}
\hline Model & Prior $P_{\text {planet }}$ & GP kernel & $\Delta \ln Z$ \\
\hline $1 \mathrm{pl}$ & $\mathcal{N}_{\mathrm{b}}\left(55.7,0.5^{2}\right)$ & $\ldots$ & 19.93 \\
\hline $2 \mathrm{pl}$ & $\begin{array}{l}\mathcal{N}_{\mathrm{b}}\left(3.931,0.001^{2}\right) \\
\mathcal{N}_{\mathrm{c}}\left(55.7,0.5^{2}\right)\end{array}$ & $\cdots$ & 15.51 \\
\hline $3 \mathrm{pl}$ & $\begin{array}{l}\mathcal{N}_{\mathrm{b}}\left(3.931,0.001^{2}\right) \\
\mathcal{N}_{\mathrm{c}}\left(9.1,0.1^{2}\right) \\
\mathcal{N}_{\mathrm{d}}\left(55.7,0.5^{2}\right)\end{array}$ & $\cdots$ & 9.03 \\
\hline $4 \mathrm{pl}$ & $\begin{array}{l}\mathcal{N}_{\mathrm{b}}\left(3.931,0.001^{2}\right) \\
\mathcal{N}_{\mathrm{c}}\left(9.1,0.1^{2}\right) \\
\mathcal{N}_{\mathrm{d}}\left(55.7,0.5^{2}\right) \\
\mathcal{N}_{\mathrm{e}}\left(87.3,0.5^{2}\right)\end{array}$ & $\ldots$ & -2.15 \\
\hline 1pl+GPexp & $\mathcal{N}_{\mathrm{b}}\left(3.931,0.001^{2}\right)$ & $\operatorname{Exp}^{(a)}$ & 19.51 \\
\hline 2pl+GPexp & $\begin{array}{l}\mathcal{N}_{\mathrm{b}}\left(3.931,0.001^{2}\right) \\
\mathcal{N}_{\mathrm{c}}\left(9.1,0.1^{2}\right)\end{array}$ & $\operatorname{Exp}^{(a)}$ & 9.29 \\
\hline 3pl+GPexp & $\begin{array}{l}\mathcal{N}_{\mathrm{b}}\left(3.931,0.001^{2}\right) \\
\mathcal{N}_{\mathrm{c}}\left(9.1,0.1^{2}\right) \\
\mathcal{N}_{\mathrm{d}}\left(55.7,0.5^{2}\right)\end{array}$ & $\operatorname{Exp}^{(a)}$ & 0.00 \\
\hline 4pl+GPexp & $\begin{array}{l}\mathcal{N}_{\mathrm{b}}\left(3.931,0.001^{2}\right) \\
\mathcal{N}_{\mathrm{c}}\left(9.1,0.1^{2}\right) \\
\mathcal{N}_{\mathrm{d}}\left(55.7,0.5^{2}\right) \\
\mathcal{N}_{\mathrm{e}}\left(87.3,0.5^{2}\right)\end{array}$ & $\operatorname{Exp}^{(a)}$ & -0.95 \\
\hline $1 \mathrm{pl}+\mathrm{GPess}$ & $\mathcal{N}_{\mathrm{b}}\left(3.931,0.001^{2}\right)$ & $\operatorname{ExpSinSq}^{(b)}$ & 18.06 \\
\hline 2pl+GPess & $\begin{array}{l}\mathcal{N}_{\mathrm{b}}\left(3.931,0.001^{2}\right) \\
\mathcal{N}_{\mathrm{c}}\left(9.1,0.1^{2}\right)\end{array}$ & $\operatorname{ExpSinSq}^{(b)}$ & 6.32 \\
\hline 3pl+GPess & $\begin{array}{l}\mathcal{N}_{\mathrm{b}}\left(3.931,0.001^{2}\right) \\
\mathcal{N}_{\mathrm{c}}\left(9.1,0.1^{2}\right) \\
\mathcal{N}_{\mathrm{d}}\left(55.7,0.5^{2}\right)\end{array}$ & $\operatorname{ExpSinSq}^{(b)}$ & -1.59 \\
\hline 4pl+GPess & $\begin{array}{l}\mathcal{N}_{\mathrm{b}}\left(3.931,0.001^{2}\right) \\
\mathcal{N}_{\mathrm{c}}\left(9.1,0.1^{2}\right) \\
\mathcal{N}_{\mathrm{d}}\left(55.7,0.5^{2}\right) \\
\mathcal{N}_{\mathrm{e}}\left(87.3,0.5^{2}\right)\end{array}$ & $\operatorname{ExpSinSq}^{(b)}$ & 3.11 \\
\hline
\end{tabular}

Notes. The prior label $\mathcal{N}$ represents a normal distribution. The final model used for the joint fit is marked in boldface (see Sect. 5.2.2 for details about the selection of the final model). ${ }^{(a)}$ Simple exponential kernel of the form $k_{i, j}=\sigma_{\mathrm{GP}, \mathrm{RV}}^{2} \exp \left(-\left|t_{i}-t_{j}\right| \mid\right.$ $\left.T_{\mathrm{GP}, \mathrm{RV}}\right){ }^{(b)}$ Exponential-sine-squared kernel of the form $k_{i, j}=\sigma_{\mathrm{GP}, \mathrm{RV}}^{2}$ $\exp \left(-\alpha_{\mathrm{GP}, \mathrm{RV}}\left(t_{i}-t_{j}\right)^{2}-\Gamma_{\mathrm{GP}, \mathrm{RV}} \sin ^{2}\left[\frac{\pi\left|t_{i}-t_{j}\right|}{P_{\mathrm{rot}, \mathrm{GP}, \mathrm{RV}}}\right]\right)$ with a uniform prior in $P_{\text {rot;GP,RV }}$ ranging from 30 to $100 \mathrm{~d}$.

of the six individual RV datasets and assumed circular orbits. We also considered eccentric orbits but found the circular model fits to have comparable log evidence and be computationally less expensive.

The first set of models $(1 \mathrm{pl}, 2 \mathrm{pl}, 3 \mathrm{pl}, 4 \mathrm{pl})$ treats the signals found in the periodogram analysis as Keplerian circular orbits. The preferred model is clearly the four-planet one, with a minimum $\Delta \ln Z>11$ with respect to the others. This model is also the one with the highest evidence in our analysis. The three signals at 3.93, 9.1, and $55.7 \mathrm{~d}$ have eccentricities compatible with zero. However, the derived eccentricity for the $87.3 \mathrm{~d}$ signal is substantially high $(e \sim 0.4)$. We notice that the RV phase-folded curve to the $87.3 \mathrm{~d}$ signal is not homogeneously sampled, with very few RV points covering both quadratures, which could explain the relatively high eccentric behavior derived for the $87.3 \mathrm{~d}$ signal.

To test the planetary nature of the signals, we also tried more complex models using GP regression to account for correlated noise. The explicit mathematical form of the GP kernels can be found in the notes to Table 3 . We first employed a quasiperiodic exponential-sine-squared kernel (GPess) using a wide prior for the period term. In doing this, we can evaluate the preference for Keplerian signals over correlated periodic noise in the data, especially focusing on modeling the dubious $87.3 \mathrm{~d}$ periodicity. Even though the posterior distribution does not show any interesting signals, a periodogram of the GP component of the 3 pl+GPess model - obtained by substracting the median Keplerian model to our full median $3 \mathrm{pl}+\mathrm{GPess}$ model - reveals that the $87.3 \mathrm{~d}$ periodicity is the main component. However, if we consider the $87.3 \mathrm{~d}$ as a Keplerian along with the other three periodicities and a GPess kernel to account for the residual noise seen in Fig. 4e, it yields worse results in terms of model log evidence. Besides, a simpler exponential kernel (GPexp) for the GP with a three-Keplerian model gives comparable results to the $3 \mathrm{pl}+\mathrm{GPess}$ model. This indicates that the exponential kernel is sufficient in accounting for the stochastic behavior of the data. Additionally, we tried a GPess kernel with a normal prior in $P_{\text {rot;GP,RV }}$ centered at the $77.8 \mathrm{~d}$ rotational period of the star derived in Sect. 4.2. In that case, the results are worse or equivalent to the GPexp kernel case, meaning that the GP model did not catch any clear periodicity. This suggests also that the stellar spots do not imprint any modulation in the RVs, which is in line with the absence of peaks around $78 \mathrm{~d}$ in the periodograms of Fig. 4.

Given the $\log$ evidence of the different models in Table 3, 4pl, 3pl+GPexp, and 3pl+GPess are statistically equivalent. Although $4 \mathrm{pl}$ is weakly favored in terms of $\ln Z$, the fact that the derived orbit of the $87.3 \mathrm{~d}$ signal is much more eccentric than the others and the phase is not well covered by our measurements withdraw us from firmly claiming the signal as of planetary nature. Further observations of GJ 357 will help shed light on the true nature of this signal and further potential candidates. Therefore, for the final joint fit we consider the model with three Keplerians and an exponential GP to be the simplest model that best explains the current data present.

\subsubsection{Joint fit}

To obtain the most precise parameters of the GJ 357 system, we performed a joint analysis of the TESS and LCO photometry and Doppler data using juliet, of the model 3pl+GPexp from our RV-only analysis in Sect. 5.2.2. In this way, we simultaneously constrain all the parameters for the transiting planet GJ $357 \mathrm{~b}$, the planet candidates at $9.12 \mathrm{~d}$ and $55.7 \mathrm{~d}$, and the correlated noise seen in the RV data with an exponential GP kernel. To optimize the computational time, we narrowed down our priors based on the analyses from the sections above, but we kept them wide enough to fully sample the posterior distribution of the quantities of interest. Our choice of the priors for each parameter in the joint analysis of the 3pl+GPexp model can be found in Table A.1.

The posterior distribution of the parameters of our best-fit joint model are presented in Table 4 . We also ran an eccentric version of the joint $3 \mathrm{pl}+\mathrm{GPexp}$ model, but the circular fit model was strongly favored $(\Delta \ln Z>8)$ and thus we only show the circular results in Table 4. The corresponding modeling of the data based on these posteriors is shown in Fig. 5 and the derived 

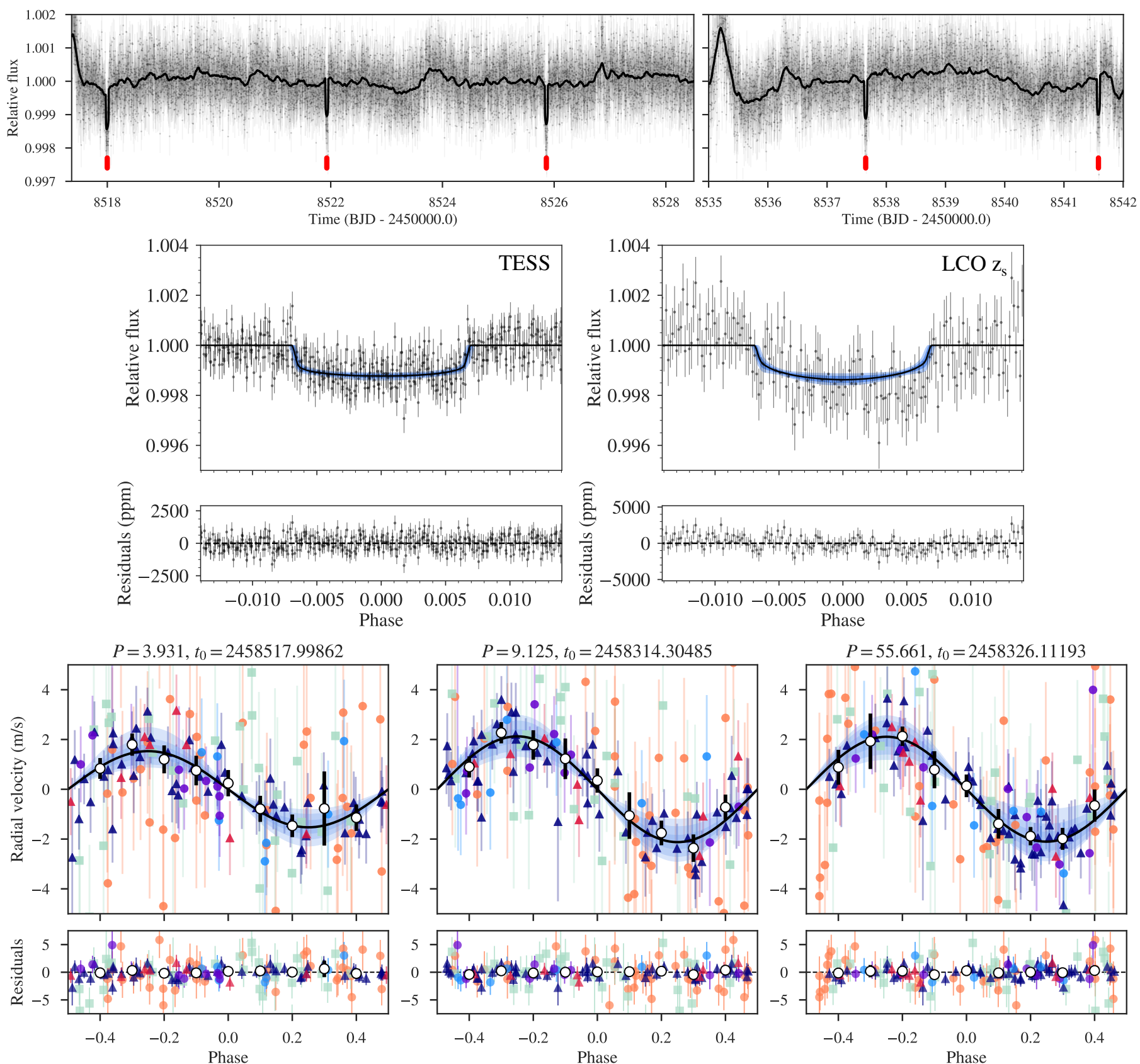

Fig. 5. Results from the joint fit of the best model 3pl+GPexp. Top panel: TESS photometry time series (gray points with error bars) along with the best-fit model (solid black line) from our joint modeling. This best-fit model includes an exponential GP used to account for the evident trends as well as for a transit model. Individual transits of GJ $357 \mathrm{~b}$ are indicated with red ticks. Middle panel: TESS photometry (left) and LCO photometry (right) phase-folded to the $3.93 \mathrm{~d}$ period of GJ $357 \mathrm{~b}$ along with best-fit transit model from the joint fit. The GP fitted to the photometry has been removed. Bottom panel: RVs phase-folded to the period of the three confirmed planets (GJ 357 b, left; GJ 357 c, center; GJ 357 d, right). RV data come from HIRES (orange circles), UVES (light green squares), HARPS (navy blue triangles), PFSpre (light blue circles), PFSpost (red triangles), and CARMENES (purple circles). The GP fitted to the RV dataset has been removed. White circles show binned datapoints in phase for visualisation. The error bars of both photometry and RV data include their corresponding jitter.

physical parameters of the system are presented in Table 5. The $\mathrm{RV}$ time series is shown for completeness in Fig. B.1.

\section{Discussion}

The GJ 357 system consists of one transiting Earth-sized planet in a $3.93 \mathrm{~d}$ orbit, namely GJ $357 \mathrm{~b}$, with a radius of $R_{\mathrm{b}}=1.217 \pm 0.084 R_{\oplus}$, a mass of $M_{\mathrm{b}}=1.84 \pm 0.31 M_{\oplus}$, and a density of $\rho_{\mathrm{b}}=5.6_{-1.3}^{+1.7} \mathrm{~g} \mathrm{~cm}^{-3}$; and two additional planets, namely GJ $357 \mathrm{c}$, with a minimum mass of $M_{\mathrm{c}}=3.40 \pm 0.46 M_{\oplus}$ in a $9.12 \mathrm{~d}$ orbit, and GJ $357 \mathrm{~d}$, with a minimum mass of $M_{\mathrm{c}}=6.1 \pm 1.0 M_{\oplus}$ in a $55.7 \mathrm{~d}$ orbit. The modulations from the GP model have an amplitude of $2.66 \mathrm{~m} \mathrm{~s}^{-1}$ and account for the short timescale of the stochastic variations and the dubious $87.3 \mathrm{~d}$ signal.

\subsection{Searching for transits of planets $c$ and $d$}

Although in Sects. 2.1 and 5.2.1 we looked for additional transit features in the TESS light curve, but could not find any, 
Table 4. Posterior parameters of the final joint fit obtained for GJ 357 b, c, and d, using juliet.

\begin{tabular}{|c|c|c|c|}
\hline Parameter ${ }^{(a)}$ & GJ 357 b & GJ 357 c & GJ $357 \mathrm{~d}$ \\
\hline \multicolumn{4}{|c|}{ Stellar parameters } \\
\hline$\rho_{\star}\left(\mathrm{kg} \mathrm{m}^{-3}\right)$ & & $0.0_{-1600}^{+1400}$ & \\
\hline
\end{tabular}

$P(\mathrm{~d})$
$t_{0}^{(b)}$
$r_{1}$
$r_{2}$
$K\left(\mathrm{~m} \mathrm{~s}^{-1}\right)$

$3.93072_{-0.00006}^{+0.00008}$
$8517.99862_{-0.00038}^{+0.00039}$
$0.56_{-0.09}^{+0.07}$
$0.0331_{-0.0009}^{+0.0009}$
$1.52_{-0.25}^{+0.25}$

$9.1247_{-0.0010}^{+0.0011}$

$8314.30_{-0.38}^{+0.42}$

$55.661_{-0.055}^{+0.055}$

$8326.1_{-3.8}^{+3.9}$

...

...

...

$2.13_{-0.28}^{+0.28}$

$2.09_{-0.35}^{+0.34}$

Photometry parameters

$M_{\mathrm{TESS}}(\mathrm{ppm})$
$M_{\mathrm{LCO}}(\mathrm{ppm})$
$\sigma_{\mathrm{TESS}}(\mathrm{ppm})$
$\sigma_{\mathrm{LCO}}(\mathrm{ppm})$
$q_{1, \mathrm{TESS}}$
$q_{2, \mathrm{TESS}}$

$q_{1, \mathrm{LCO}}$

$\mu_{\text {HIRES }}\left(\mathrm{m} \mathrm{s}^{-1}\right)$
$\sigma_{\text {HIRES }}\left(\mathrm{m} \mathrm{s}^{-1}\right)$
$\mu_{\text {UVES }}\left(\mathrm{m} \mathrm{s}^{-1}\right)$
$\sigma_{\text {UVES }}\left(\mathrm{m} \mathrm{s}^{-1}\right)$
$\mu_{\text {HARPS }}\left(\mathrm{m} \mathrm{s}^{-1}\right)$
$\sigma_{\text {HARPS }}\left(\mathrm{m} \mathrm{s}^{-1}\right)$
$\mu_{\text {PFSpre }}\left(\mathrm{m} \mathrm{s}^{-1}\right)$
$\sigma_{\text {PFSpre }}\left(\mathrm{m} \mathrm{s}^{-1}\right)$
$\mu_{\text {PFSpost }}\left(\mathrm{m} \mathrm{s}^{-1}\right)$
$\sigma_{\text {PFSpost }}\left(\mathrm{m} \mathrm{s}^{-1}\right)$
$\mu_{\text {CARM }}\left(\mathrm{m} \mathrm{s}^{-1}\right)$
$\sigma_{\text {CARM }}\left(\mathrm{m} \mathrm{s}^{-1}\right)$

$\sigma_{\mathrm{GP}, \mathrm{TESS}}(\mathrm{ppm})$

$T_{\mathrm{GP}, \mathrm{TESS}}(\mathrm{d})$

$\sigma_{\mathrm{GP}, \mathrm{RV}}\left(\mathrm{m} \mathrm{s}^{-1}\right)$

$T_{\mathrm{GP}, \mathrm{RV}}(\mathrm{d})$
Table 5. Derived planetary parameters obtained for GJ $357 \mathrm{~b}$, c, and d using the posterior values from Table 4.

\begin{tabular}{lccc}
\hline \hline Parameter $^{(a)}$ & GJ $357 \mathrm{~b}$ & GJ 357 c & GJ 357 d \\
\hline \multicolumn{4}{c}{ Derived transit parameters } \\
$p=R_{\mathrm{p}} / R_{\star}$ & $0.0331_{-0.0009}^{+0.0009}$ & $\ldots$ & $\ldots$ \\
$b=\left(a / R_{\star}\right) \cos i_{\mathrm{p}}$ & $0.34_{-0.14}^{+0.10}$ & $\ldots$ & $\ldots$ \\
$a / R_{\star}$ & $22.31_{-0.90}^{+0.76}$ & $\ldots$ & $\ldots$ \\
$i_{p}(\mathrm{deg})$ & $89.12_{-0.31}^{+0.37}$ & $\ldots$ & $\ldots$ \\
$u_{1}{ }^{(b)}$ & $0.27_{-0.17}^{+0.24}$ & $\ldots$ & $\ldots$ \\
$u_{2}{ }^{(b)}$ & $0.14_{-0.24}^{+0.29}$ & $\ldots$ & $\ldots$ \\
$t_{T}(\mathrm{~h})$ & $1.53_{-0.11}^{+0.12}$ & $\ldots$ & $\ldots$
\end{tabular}

\section{Derived physical parameters}

$$
\begin{aligned}
& M_{\mathrm{p}}\left(M_{\oplus}\right)^{(c)} \\
& R_{\mathrm{p}}\left(R_{\oplus}\right) \\
& \rho_{\mathrm{p}}\left(\mathrm{g} \mathrm{cm}^{-3}\right) \\
& g_{\mathrm{p}}\left(\mathrm{m} \mathrm{s}^{-2}\right) \\
& a_{\mathrm{p}}(\mathrm{au}) \\
& T_{\mathrm{eq}}(\mathrm{K})^{(d)} \\
& S\left(S_{\oplus}\right)
\end{aligned}
$$

$$
\begin{array}{cc}
>3.40_{-0.46}^{+0.46} & >6.1_{-1.0}^{+1.0} \\
\ldots & \ldots \\
\ldots & \ldots \\
\ldots & \ldots \\
0.061_{-0.004}^{+0.004} & 0.204_{-0.015}^{+0.015} \\
401.2_{-10.7}^{+10.8} & 219.6_{-5.9}^{+5.9} \\
4.45_{-0.14}^{+0.14} & 0.38_{-0.01}^{+0.01}
\end{array}
$$

Notes. ${ }^{(a)}$ Error bars denote the $68 \%$ posterior credibility intervals. ${ }^{(b)}$ Derived only from the TESS light curve. ${ }^{(c)}$ The masses for GJ $357 \mathrm{c}$ and GJ $357 \mathrm{~d}$ are a lower limit $\left(M_{\mathrm{p}} \sin i\right)$ since they are detected in the RV data only. ${ }^{(d)}$ Equilibrium temperatures were calculated assuming zero Bond albedo.

any possible transit of GJ $357 \mathrm{~d}$ would have been missed by TESS observations, according to the predicted transit epoch from the RVs fits. With the current data the uncertainty in the ephemeris is on the order of days, however, additional RVs could improve the precision on the period and phase determination enough to allow a transit search. The a priori transit probability is only $0.8 \%$, but this transit search is doable by CHEOPS (Broeg et al. 2013) since the star will fall in its $50 \mathrm{~d}$ observability window.

As in other planetary systems recently revealed by TESS (e.g., Espinoza et al. 2019) or Kepler/K2 (e.g., Quinn et al. 2015; Buchhave et al. 2016; Otor et al. 2016; Christiansen et al. 2017; Gandolfi et al. 2017; Cloutier et al. 2017), non-transiting planets in these transit-detected systems expand our understanding of the system architecture. In those works, after the modeling of the transiting planet detected in the space-based photometry, further signals appear in the RV data that would not have been significant otherwise. This emphasizes the importance of long, systematic, and precise ground-based searches for planets around bright stars and the relevance of archival public data.

\subsection{System architecture}

We determine that the transiting planet GJ $357 \mathrm{~b}$ has a mass of $M_{\mathrm{b}}=1.84 \pm 0.31 M_{\oplus}$ and a radius of $R_{\mathrm{b}}=1.217 \pm 0.084 R_{\oplus}$, which corresponds to a bulk density of $\rho_{\mathrm{b}}=5.6_{-1.3}^{+1.7} \mathrm{~g} \mathrm{~cm}^{-3}$. Figure 6 shows masses and radii of all confirmed planets whose precision in both parameters is better than 30\%. GJ $357 \mathrm{~b}$ joins the very small group of Earth-sized and Earth-mass planets orbiting $\mathrm{M}$ dwarfs discussed in the introduction. The bulk 


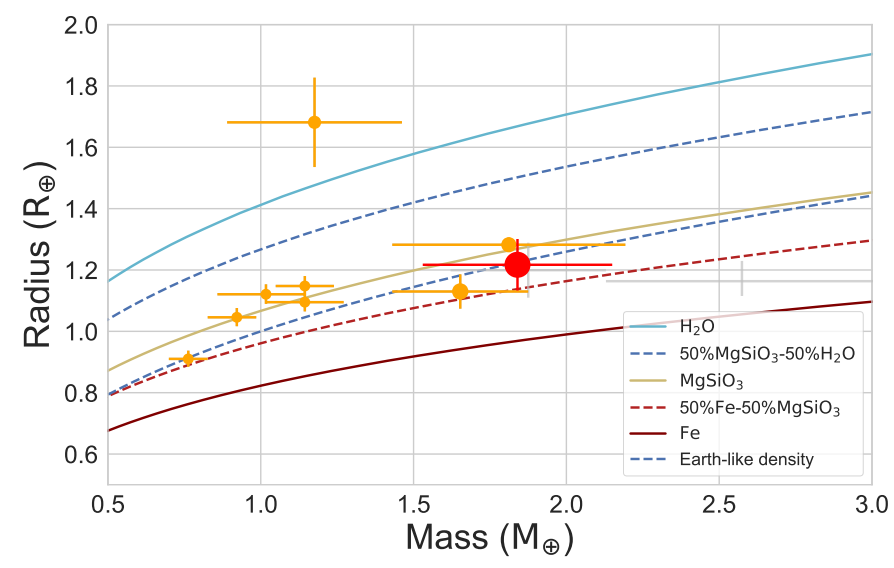

Fig. 6. Mass-radius diagram for all known transiting planets with masses between $0.5-3 M_{\oplus}$ and radii $0.5-2 R_{\oplus}$ determined with a precision better than $30 \%$. GJ $357 \mathrm{~b}$ is shown in red. Planets orbiting around late-type stars $\left(T_{\text {eff }}<4000 \mathrm{~K}\right)$ are shown in orange, otherwise gray. The size of the orange datapoints is inversely proportional to the magnitude of their host star in the $J$-band. Data are taken from the TEPCat database of well-characterized planets (Southworth 2011). Theoretical models for the planet's internal composition are taken from Zeng et al. (2016).

density we measure for GJ 357 b overlaps with the $30 \% \mathrm{Fe}$ and $70 \% \mathrm{MgSiO}_{3}$ mass-radius curve as calculated by Zeng et al. (2016).

We derive a minimum mass for GJ $357 \mathrm{c}$ of $M_{\mathrm{c}} \sin i_{\mathrm{c}}=$ $3.40 \pm 0.46 M_{\oplus}$, which falls between the vaguely defined mass range for Earth- and super-Earth-like planets. Since we determine only a lower limit for $M_{\mathrm{c}}$, it is possible that this planet falls into the super-Earth category joining the group of planets that make up the lower radius bump in the bimodal distribution of small planets found with Kepler (Fulton et al. 2017; Van Eylen et al. 2018).

The derived minimum mass of GJ $357 \mathrm{~d}$ is $M_{\mathrm{d}} \sin i_{\mathrm{d}}=$ $6.1 \pm 1.0 M_{\oplus}$. However, a prediction of its bulk composition is not straightforward since both super-Earth $\left(1<R<2 R_{\oplus}\right)$ and mini-Neptune $\left(2<R<4 R_{\oplus}\right)$ exoplanets encompass this range of masses. As an example of this dichotomy, Kepler-68 b (Gilliland et al. 2013, $R=2.33 \pm 0.02 R_{\oplus}$ ) and Kepler-406 b (Marcy et al. $2014, R=1.43 \pm 0.03 R_{\oplus}$ ) have similar masses of $M \sim 6.0 M_{\oplus}$ and $M \sim 6.3 M_{\oplus}$, respectively, but their compositions differ significantly (between rocky and gaseous for Kepler-68 b, and purely rocky for Kepler-406 b).

We note that the planetary system of GJ 357 is quite similar to that of GJ 1132 (Berta-Thompson et al. 2015; Bonfils et al. 2018). In both cases we find a similar bulk density of the inner planet of $\sim 6 \mathrm{~g} \mathrm{~cm}^{-3}$ (see orange datapoint down left of GJ 357 b in Fig. 6) and a second non-transiting planet with an orbital period around $9 \mathrm{~d}$. Furthermore, the long-term trends found with GJ 1132 could indicate the presence of one or more outer, more massive planets, which would then be comparable to the existence of planet $d$ in GJ 357.

\subsection{Dynamics and TTV analysis}

While a detailed characterization of the dynamical properties of the potential planetary system is beyond the scope of this paper, we nevertheless started to investigate its properties using Systemic (Meschiari et al. 2009). Given the fact that TESS could only cover five transits, the detection of transit timing variations (TTV) would only be possible in a system with more massive planets or in a first order resonance like, for example, in Kepler-87 (Ofir et al. 2014). The inner pair of planets is, however, close to a 7:3 period commensurability. The dynamical interactions are small but, depending on the initial eccentricities, the system may undergo significant exchange of angular momentum, but on very long timescales of $\sim 500 \mathrm{yr}$, which are clearly too long to be detectable in the currently available RV measurements.

Since we cannot detect a transit for planet c, its orbit could be inclined with respect to planet $b$. The inclination of planet $c$ only needs to be $<88.5 \pm 0.1 \mathrm{deg}$ for a non-detection, meaning a very gentle tilt with respect to planet $\mathrm{b}\left(i_{\mathrm{b}}=89.12_{-0.31}^{+0.37} \mathrm{deg}\right)$ would be enough to miss it. For planet $d$ to transit, doing this same exercise implies one would need inclinations larger than about $89.55+$ $-0.04 \mathrm{deg}$, which is a $1 \mathrm{deg}$ difference in mutual inclination with planet c, and a 0.4 deg difference with the transiting planet $b$. Since multi-planet systems in general have mutual inclinations within $\sim 2$ deg from each other (Dai et al. 2018), there is still room for planet $d$ to be a transiting planet. For initially low eccentric configurations, the inclination probably cannot be constrained from dynamics, because preliminary tests show that the system is stable even for a low inclination for planet $\mathrm{c}$ of $i_{\mathrm{c}}=10 \mathrm{deg}$. At large mutual inclinations ( $30 \mathrm{deg}$ ), the mutual torque is significant and planet $b$ would drift slowly out of a transiting configuration. This effect, however, reduces significantly with a lower mutual inclination, when small mutual tilts result in torques that could bring planet $\mathrm{c}$ or $\mathrm{d}$ into transit or planet $\mathrm{b}$ out of transit. A long-term monitoring of planet $b$ could show or constraint inclination changes from transit duration or depth variations (TDVs).

We carried out a more in-depth search for TTVs using PYTRANSIT (Parviainen 2015). The approach models the near vicinity of each transit $(4.8 \mathrm{~h}$ around the expected transit center based on the linear ephemeris) as a product of a transit model and a flux baseline made of $n_{\mathrm{L}}$ Legendre polynomials. The transits are modeled jointly, and parametrized by the stellar density, impact parameter, planet-star area ratio, two quadratic limb darkening coefficients, an independent transit center for each transit, and $n_{\mathrm{L}}$ Legendre polynomial coefficients for each transit (modeling the baseline as a sum of polynomials rather than, for example, a Gaussian process, is still feasible given the small number of transits). The analysis results in an estimate of the model posterior distribution, where the independent parameter estimates are based on their marginal posterior distributions. The uncertainty in the transit center estimates - calculated as $0.5 \times\left(t_{84}-t_{16}\right)$, where $t_{16}$ and $t_{84}$ are the 16th and 84th transit center posterior percentiles - varies from 1.5 to $4 \mathrm{~min}$, and no significant deviations from the linear ephemeris can be detected, in agreement with our previous estimate.

\subsection{Formation history}

The predominant formation channel to build terrestrial planets is the core accretion scenario, where a solid core is formed before the accretion of an atmosphere sets in (Mordasini et al. 2012). This scenario involves several stages: first, dust grows into larger particles that experience vertical settling and radial drift, commonly referred to as "pebbles" (Birnstiel et al. 2012). They can undergo gravitational collapse into $\sim 100 \mathrm{~km}$ sized planetesimals wherever a local concentration exceeds a level set by the disk turbulence (Johansen et al. 2007; Lenz et al. 2019). These planetesimals can form planetary embryos of roughly Moon size via mutual collisions (Levison et al. 2015), at which point an accretion of further planetesimals and, even more importantly, of drifting pebbles can lead to further growth 
(Ormel \& Klahr 2010). Pebble accretion is thus very powerful in quickly forming the cores of gas giants (Klahr \& Bodenheimer 2006; Lambrechts et al. 2014), but usually fails to produce terrestrial planets akin to the inner solar system due to its high efficiency. To explain the low final mass of such planets, a mechanism that stops further accretion of solids is needed.

One way to inhibit pebble accretion is to cut the supply of solid material by another planet that forms further out earlier than or concurrently with the inner planets. Such a companion would act as a sink for the influx of material that would otherwise be available to build inner planets. This is achieved when the outer planet reaches a critical mass where the fraction of the pebble flux accreted by the planet

$\epsilon_{\mathrm{PA}} \approx 0.1 \times\left(\frac{q}{10^{-5}}\right)^{\frac{2}{3}}, \quad$ with $q=\frac{M_{\mathrm{Planet}}}{M_{\star}}$,

approaches unity (Ormel et al. 2017). With $q=3 \times 10^{-5}$ and $\epsilon_{\mathrm{PA}} \approx 0.2$, GJ $357 \mathrm{c}$ could have efficiently absorbed pebbles that would otherwise have reached GJ 357 b. Likewise, GJ 357 d reached a pebble accretion efficiency of $\epsilon_{\mathrm{PA}} \approx 0.3$ and could have starved the inner two planets of further accretion of pebbles. In this scenario, the planets must have formed outside-in and one would expect one or several additional planets of at least the mass of GJ $357 \mathrm{~d}$ further out. Such a hypothetical GJ 357 e again should have reached high pebble accretion efficiencies before its inner siblings. This is quite feasible, since the conditions for fast embryo formation are very favorable just outside the ice line of the protoplanetary disk, where the recondensation of vapor leads to a large abundance of planetesimals (Stevenson \& Lunine 1988; Cuzzi \& Zahnle 2004; Ciesla \& Cuzzi 2006; Schoonenberg \& Ormel 2017). We used the minimum masses of GJ $357 \mathrm{c}$ and GJ $357 \mathrm{~d}$ for these estimates. Thus, the efficiencies we calculated should be considered conservative, making this mechanism even more robust.

However, timing is key in order to stop the supply of solid material at the right time. The window is only open for $\sim 10^{5} \mathrm{yr}$, which corresponds to the timescale for growing from a roughly Earth-sized planet to a super-Earth (e.g., Bitsch et al. 2019).

A scenario with less stringent assumptions is one where an inner planet grows to its own pebble isolation mass, which can be approximated as

$M_{\text {iso }} \approx h^{3} M_{\star}$

with the local disk aspect ratio $h$ (Ormel et al. 2017). Reaching $M_{\text {iso }}$, the planet locally modifies the radial gas pressure gradient such that the inward drift of pebble-sized particles stops, starving itself and the inner system of solid material. Assuming the current orbit of GJ $357 \mathrm{~b}$ and an $M_{\text {iso }}$ equal to the planetary mass inferred in our study, Eq. (3) yields a local disk aspect ratio of 0.025 , which is a reasonable value in the inner disk. Similarly, the minimum masses of GJ $357 \mathrm{c}$ and GJ $357 \mathrm{~d}$ give $h \approx 0.031$ and $h \approx 0.038$, respectively, which is again consistent with estimated disk scale heights in the literature (e.g., Chiang \& Goldreich 1997; Ormel et al. 2017).

Given the significant assumptions needed to explain the emergence of both planets by a cut-off of pebble flux in the system, the second scenario is favored. If pebble accretion is the dominating mechanism to form planetary embryos in the system, then GJ 357 b-d stopped growing when they reached their respective pebble isolation masses. However, a hypothetical future discovery of a more massive planet further out might shift the balance again towards shielding by this outer planet.

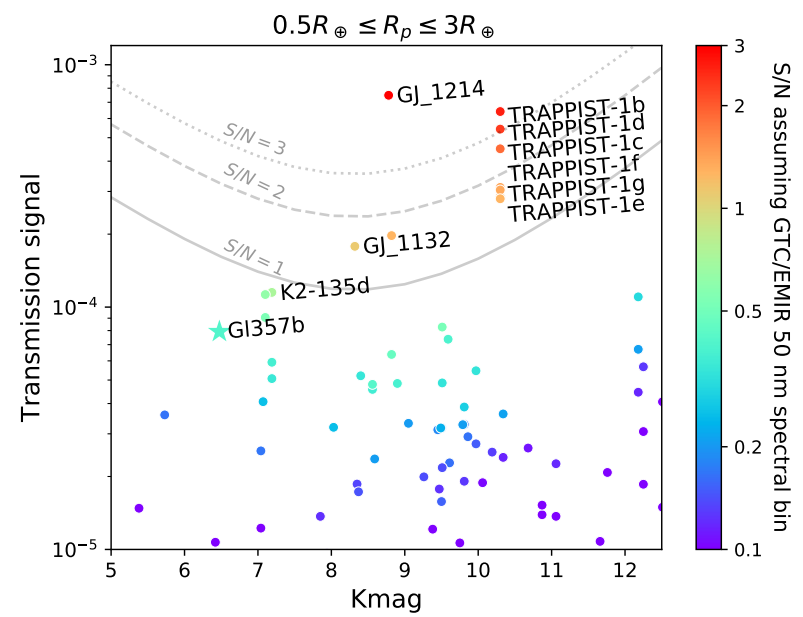

Fig. 7. Expected primary transit transmission signal per scale height plotted against the $K$-band magnitude of GJ 357 b (star) and all know planets (dots) with mass measurements and with radius between 0.5 and $3 R_{\oplus}$. The color scale provides the expected $\mathrm{S} / \mathrm{N}$ for a single transit assuming the use of a ten-meter telescope and $50 \mathrm{~nm}$ wavelength integration bins. The gray lines indicate the pattern of the $\mathrm{S} / \mathrm{N}$ assuming a single transit duration of $3 \mathrm{~h}$. The relative $\mathrm{S} / \mathrm{N}$ is maintained when extrapolated to other instrumentation. A few benchmark targets for JWST transmission spectroscopy studies are labeled.

\subsection{Atmospheric characterization and habitability}

The integrated stellar flux that hits the top of an Earth-like planet's atmosphere from a cool red star warms the planet more efficiently than the same integrated flux from a hot blue star. This is partly due to the effectiveness of the Rayleigh scattering in an atmosphere mostly composed of $\mathrm{N}_{2}-\mathrm{H}_{2} \mathrm{O}-\mathrm{CO}_{2}$, which decreases at longer wavelengths, together with the increased near-IR absorption by $\mathrm{H}_{2} \mathrm{O}$ and $\mathrm{CO}_{2}$.

Planets GJ 357 b and GJ 357 c receive about 13 times and 4.4 times the Earth's irradiation $\left(S_{\oplus}\right)$, respectively. Venus in comparison receives about $1.7 S_{\oplus}$. Thus, both planets should have undergone a runaway greenhouse stage as proposed for Venus' evolution. Due to its incident flux level, GJ $357 \mathrm{c}$ is located closer to the star than the inner edge of the empirical habitable zone as defined in Kasting et al. (1993) and Kopparapu et al. (2014). On the other hand, GJ $357 \mathrm{~d}$ receives an irradiation of $0.38 S_{\oplus}$, which places it inside the habitable zone (as defined above), in a location comparable to Mars in the solar sytem, making it a very interesting target for further atmospheric observations.

Atmospheric characterization of exoplanets is difficult because of the high contrast ratio between a planet and its host star. While atmospheres of Earth and super-Earth planets are still outside our technical capabilities, upcoming space missions such as the James Webb Space Telescope (JWST) and the extremely large ground-based telescopes (ELTs) will open this possibility for a selected group of rocky planets offering the most favorable conditions.

The star GJ 357 is one of the brightest M dwarfs in the sky, and as such, planets orbiting it are interesting targets for follow-up characterization. To illustrate this fact, in Fig. 7 we plot the expected transmission signal reachable in a single transit with a ground-based $10 \mathrm{~m}$ telescope for all known planets with mass measurements and with radius between 0.5 and $3 R_{\oplus}$. The transmission signal per scale height is defined as

$\mathrm{TS}=2 H_{\mathrm{s}} \frac{R_{\mathrm{p}}}{R_{\mathrm{s}}^{2}}$, 
where $R_{\mathrm{p}}$ and $R_{\mathrm{S}}$ are the radii of planet and star, respectively, and $H$ is the scale height

$H_{\mathrm{s}}=\frac{k_{\mathrm{B}} T_{\mathrm{eq}}}{\mu g_{\mathrm{p}}}$,

where $k_{\mathrm{B}}$ is the Boltzmann constant, $T_{\text {eq }}$ and $g_{\mathrm{p}}$ are the equilibrium temperature and surface gravity of the planet, respectively, and $\mu=2.3 \mathrm{~g} \mathrm{~mol}^{-1}$ is the mean molecular weight. The signal is calculated then as $1.8 \times$ TS where a spectral modulation of $1.8 H_{\mathrm{s}}$ is adopted (Iyer et al. 2016). This signal is an optimistic estimate, because terrestrial planets are unlikely to host an atmosphere of mean molecular weight at $2.3 \mathrm{~g} \mathrm{~mol}^{-1}$. The most favorable planets for atmospheric characterization offer a combination of a large scale height (puffiness of the atmospheres) and host star brightness, and are labeled in the figure together with GJ $357 \mathrm{~b}$.

Kempton et al. (2018) proposed a metric to select TESS (and other missions) planet candidates according to their suitability for atmospheric characterization studies. Using the mass and radius determined in this work $\left(1.84 M_{\oplus}, 1.217 R_{\oplus}\right)$, we obtained a transmission metric value of 23.4 for GJ $357 \mathrm{~b}$. For comparison, two of the most well-known planets around bright M-type stars with favorable metrics, LHS $1140 \mathrm{~b}$ and TRAPPIST-1 f, have metric values of 9.13 and 13.7, respectively. It is worth noting that out of the simulated yield of TESS terrestrial planets with $R<2 R_{\oplus}$ used in Kempton et al. (2018), in turn based on Sullivan et al. (2015) and assuming an Earth-like composition, only one had a larger metric value (28.2). Using the same reference, the emission spectroscopy metric for GJ $357 \mathrm{~b}$ is 4.1 , a modest number compared to the simulated yield of TESS planets suitable for these types of studies.

In order to assess an estimation of GJ 357 b's atmospheric signal through transmission spectroscopy, we simulated a simplified atmospheric photochemistry model for a rocky planet basing it on early Earth's temperature structure, increasing the surface temperature to be consistent with $T_{\text {eff }}=525 \mathrm{~K}$ and removing water from the atmosphere using ChemKM (Molaverdikhani, in prep.). The temperature and pressure structures of GJ 357 b's atmosphere are not modeled self consistently, as this only shows sample spectra.

Geometric mean spectra of GJ $667 \mathrm{C}\left(T_{\mathrm{eff}}=3327 \mathrm{~K}\right)$ and GJ $832\left(T_{\text {eff }}=3816 \mathrm{~K}\right)$ were considered as an estimation of GJ 357's flux in the range of X-ray to optical wavelengths. The data were obtained from the MUSCLES database (France et al. 2016). We modeled three different metallicities, $1 \times, 10 \times$, and $100 \times$ solar metallicity to explore a wider range of possibilities (Wakeford et al. 2017) and selected a temperature and atmosphere profile based on an anoxic Earth atmosphere. We selected three geological epochs, namely 2.0 Gyr (after the Great Oxygenation Event), 0.8 Gyr (after the Neoproterozoic Oxygenation Event, when multicellular life began to emerge), and the modern Earth (Kawashima \& Rugheimer 2019), to consider three different atmospheric conditions with different temperature structures. To set up the models, we used Venot et al. (2012)'s full kinetic network and an updated version of Hébrard et al. (2012)'s UV absorption cross sections and branching yields.

Synthetic sample transmission spectra for GJ $357 \mathrm{~b}$ are calculated using peti tRADTRANS (Mollière et al. 2019), shown in Fig. 8. The major opacity source in the atmosphere is mostly methane, and as expected $\mathrm{CH}_{4}$ and $\mathrm{CO}_{2}$ contribute more significantly at higher temperatures and metallicities in this class of planets (Molaverdikhani et al. 2019). Such spectral features are expected to be above JWST's noise floor; 20 ppm, 30 ppm, and $50 \mathrm{ppm}$, for NIRISS SOSS, NIRCam grism, and MIRI LRS,

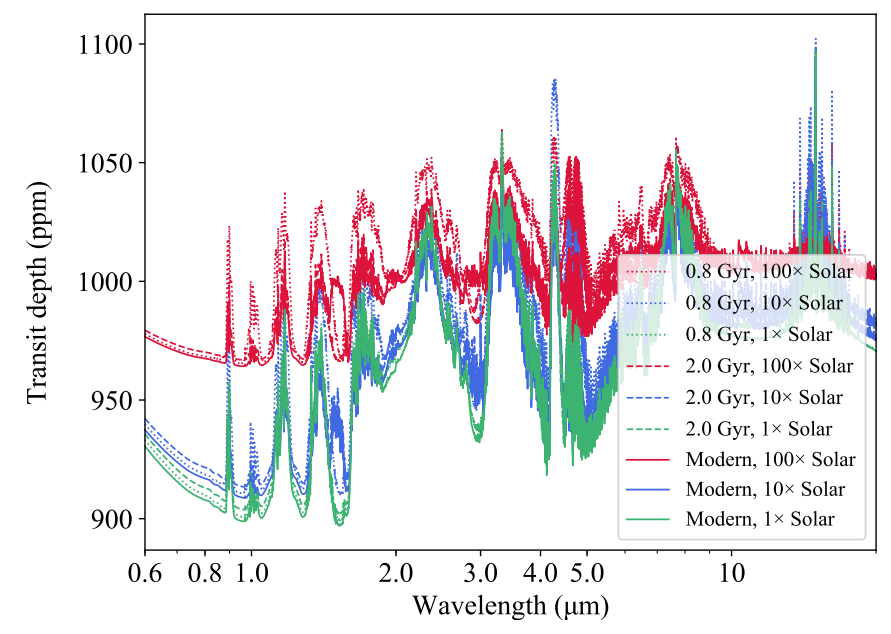

Fig. 8. Synthetic spectra of GJ $357 \mathrm{~b}$ in the JWST wavelength range. Nine transmission spectra, including three atmospheric metallicities and three temperature structures from three different Earth epochs are shown. The assumed temperature profile is the Earth's one adapted for $T_{\text {eff }}=525 \mathrm{~K}$ to resemble a hot-terminator scenario.

respectively (Greene et al. 2016). Ground-based, high-resolution spectroscopy can potentially access these strong absorption lines too. We must emphasize that these synthetic spectra are calculated under the assumption of cloud-free atmospheres. If clouds were present, the spectral features could be obscured or completely muted, resulting in a flattened transmission spectrum (Kreidberg et al. 2014).

As a final note on the atmospheric characterization of GJ 357 b, the star may be too bright in some of the JWST's observing modes, which demands careful observational planning for transmission spectroscopy. Such bright objects, however, are excellent for ground-based facilities. Louie et al. (2018) pointed out that only a few TESS planets of terrestrial size are expected to be as good or better targets for atmospheric characterization than the currently known planets. GJ $357 \mathrm{~b}$ is one of them and, although it is not in the habitable zone, in fact it could be so far the best terrestrial planet for atmospheric characterization with the upcoming JWST and ground-based ELTs.

For GJ 357 d, a rocky Earth-like composition corresponds to a $1.75 R_{\oplus}$ planet, while an ice composition would correspond to a planet radius of $2.4 R_{\oplus}$. We still do not know whether GJ $357 \mathrm{~d}$ transits its host star, however, if it did, the atmospheric signal (assuming an Earth-like composition and atmosphere) would become detectable by JWST for both NIRISS/NIRSpec (Fig. 9) and MIRI. Self-consistent models of the planet, as well as an expected atmospheric signal for GJ $357 \mathrm{~d}$, assuming a range of different compositions and atmospheres, show cool surface temperatures for Earth-like models and warm conditions for early Earth-like models. The models as well as the observable spectral features have been generated using EXO-Prime (see, e.g., Kaltenegger \& Sasselov 2010) and are discussed in detail in Kaltenegger et al. (in prep.). The code incorporates a $1 \mathrm{D}$ climate, $1 \mathrm{D}$ photochemistry, and $1 \mathrm{D}$ radiative transfer model that simulates both the effects of stellar radiation on a planetary environment and the planet's outgoing spectrum.

\section{Conclusions}

We report the discovery and confirmation of a planetary system around the bright $\mathrm{M}$ dwarf GJ 357. Data from the TESS mission revealed the first clue of this discovery by detecting the 

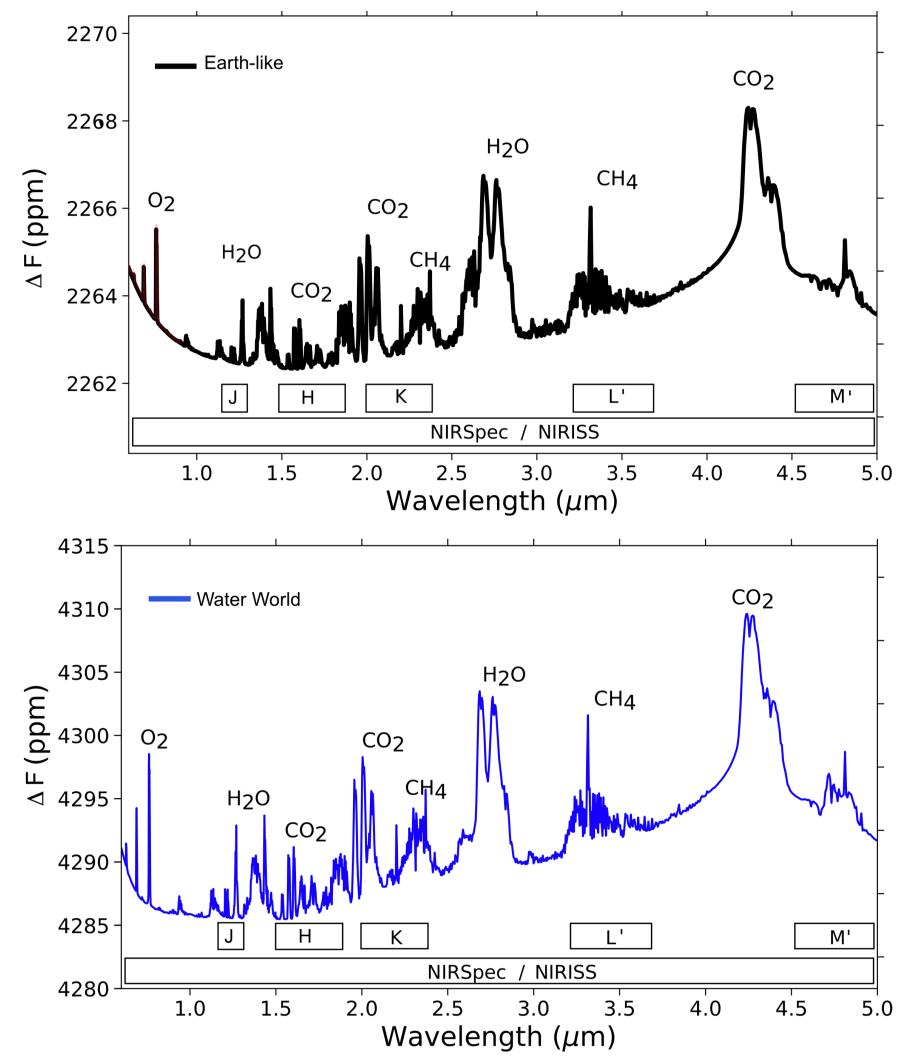

Fig. 9. Synthetic spectra for GJ $357 \mathrm{~d}$ in the JWST NIRISS/NIRSpec wavelength range for two models: Earth-like composition and atmosphere (assuming a radius of $1.75 R_{\oplus}$, black) and water world composition with Earth-like atmosphere (assuming a radius of $2.4 R_{\oplus}$,blue) as an example for detectable features.

transit signals of GJ $357 \mathrm{~b}$ through the TESS Alerts website. The availability of archival and new high-precision RV data made possible the quick confirmation of GJ $357 \mathrm{~b}$, and a search for further planet candidates in the system.

Planet GJ 357 b is a hot Earth-sized transiting planet with a mass of $1.84 \pm 0.31 M_{\oplus}$ in a $3.93 \mathrm{~d}$ orbit. The brightness of the planet's host star makes GJ 357 b one of the prime targets for future atmospheric characterization, and arguably one of the best future opportunities to characterize a terrestrial planet atmosphere with JWST and ELTs. To date, GJ $357 \mathrm{~b}$ is the nearest transiting planet to the Sun around an M dwarf and contributes to the TESS Level One Science Requirement of delivering 50 transiting small planets (with radii smaller than $4 R_{\oplus}$ ) with measured masses to the community.

Finally, there is evidence for at least two more planets, namely GJ $357 \mathrm{c}$, with a minimum mass of $3.4 \pm 0.46 M_{\oplus}$ in a $9.12 \mathrm{~d}$ orbit, and GJ $357 \mathrm{~d}$, an interesting super-Earth or subNeptune with a minimum mass of $6.1 \pm 1.0 M_{\oplus}$ in a $55.7 \mathrm{~d}$ orbit inside the habitable zone. Thus, GJ 357 adds to the growing list of TESS discoveries deserving more in-depth studies, as these systems can provide relevant information for our understanding of planet formation and evolution.

Acknowledgements. This paper includes data collected by the TESS mission Funding for the TESS mission is provided by the NASA Explorer Program. We acknowledge the use of TESS Alert data, which is currently in a beta test phase, from pipelines at the TESS Science Office and at the TESS Science Processing Operations Center. Resources supporting this work were provided by the NASA High-End Computing (HEC) Program through the NASA Advanced Supercomputing (NAS) Division at Ames Research Center for the production of the SPOC data products. This research has made use of the Exoplanet Follow-up
Observation Program website, which is operated by the California Institute of Technology, under contract with the National Aeronautics and Space Administration under the Exoplanet Exploration Program. This work has made use of data from the European Space Agency (ESA) mission Gaia (https://www. cosmos.esa.int/gaia), processed by the Gaia Data Processing and Analysis Consortium (DPAC, https://www. cosmos.esa.int/web/gaia/dpac/ consortium). Funding for the DPAC has been provided by national institutions, in particular the institutions participating in the Gaia Multilateral Agreement. CARMENES is an instrument for the Centro Astronómico Hispano-Alemán de Calar Alto (CAHA, Almería, Spain) funded by the German Max-PlanckGesellschaft (MPG), the Spanish Consejo Superior de Investigaciones Científicas (CSIC), the European Union through FEDER/ERF FICTS-2011-02 funds, and the members of the CARMENES Consortium. R. L. has received funding from the European Union's Horizon 2020 research and innovation program under the Marie Skłodowska-Curie grant agreement No. 713673 and financial support through the "la Caixa" INPhINIT Fellowship Grant LCF/BQ/IN17/11620033 for Doctoral studies at Spanish Research Centers of Excellence from "la Caixa" Banking Foundation, Barcelona, Spain. This work is partly financed by the Spanish Ministry of Economics and Competitiveness through projects ESP201680435-C2-2-R and ESP2016-80435-C2-1-R. We acknowledge support from the Deutsche Forschungsgemeinschaft under DFG Research Unit FOR2544 "Blue Planets around Red Stars", project no. QU 113/4-1, QU 113/5-1, RE 1664/14-1, DR 281/32-1, JE 701/3-1, RE 2694/4-1, the Klaus Tschira Foundation, and the Heising Simons Foundation. This work is partly supported by JSPS KAKENHI Grant Numbers JP15H02063, JP18H01265, JP18H05439, JP18H05442, and JST PRESTO Grant Number JPMJPR1775. This research has made use of the services of the ESO Science Archive Facility. Based on observations collected at the European Southern Observatory under ESO programs 072.C-0488(E), 183.C0437(A), 072.C-0495, 078.C-0829, and 173.C-0606. IRD is operated by the Astrobiology Center of the National Institutes of Natural Sciences. M.S. thanks Bertram Bitsch for stimulating discussions about pebble accretion.

\section{References}

Almenara, J. M., Astudillo-Defru, N., Bonfils, X., et al. 2015, A\&A, 581, L7 Alonso-Floriano, F. J., Morales, J. C., Caballero, J. A., et al. 2015, A\&A, 577, A128

Ambikasaran, S., Foreman-Mackey, D., Greengard, L., Hogg, D. W., \& O’Neil, M. 2015, IEEE Trans. Pattern Anal. Mach. Intell., 38, 252

Anglada-Escudé, G., Tuomi, M., Gerlach, E., et al. 2013, A\&A, 556, A126

Anglada-Escudé, G., Amado, P. J., Barnes, J., et al. 2016, Nature, 536, 437

Astudillo-Defru, N., Delfosse, X., Bonfils, X., et al. 2017, A\&A, 600, A13

Bakos, G., Noyes, R. W., Kovács, G., et al. 2004, PASP, 116, 266

Batalha, N. E., Lewis, N. K., Line, M. R., Valenti, J., \& Stevenson, K. 2018, ApJ, 856, L34

Berta-Thompson, Z. K., Irwin, J., Charbonneau, D., et al. 2015, Nature, 527, 204

Birnstiel, T., Klahr, H., \& Ercolano, B. 2012, A\&A, 539, A148

Bitsch, B., Izidoro, A., Johansen, A., et al. 2019, A\&A, 623, A88

Bonfils, X., Delfosse, X., Udry, S., et al. 2013, A\&A, 549, A109

Bonfils, X., Almenara, J. M., Cloutier, R., et al. 2018, A\&A, 618, A142

Boro Saikia, S., Marvin, C. J., Jeffers, S. V., et al. 2018, A\&A, 616, A108

Broeg, C., Fortier, A., Ehrenreich, D., et al. 2013, Eur. Phys. J. Web Conf., 47, 03005

Brown, T. M., Baliber, N., Bianco, F. B., et al. 2013, PASP, 125, 1031

Buchhave, L. A., Dressing, C. D., Dumusque, X., et al. 2016, AJ, 152, 160

Buchner, J., Georgakakis, A., Nandra, K., et al. 2014, A\&A, 564, A125

Butler, R. P., Marcy, G. W., Williams, E., et al. 1996, PASP, 108, 500

Butler, R. P., Vogt, S. S., Laughlin, G., et al. 2017, AJ, 153, 208

Charbonneau, D., Irwin, J., Nutzman, P., \& Falco, E. E. 2008, BAAS, 40, 242

Chiang, E. I., \& Goldreich, P. 1997, ApJ, 490, 368

Christiansen, J. L., Vanderburg, A., Burt, J., et al. 2017, AJ, 154, 122

Ciesla, F. J., \& Cuzzi, J. N. 2006, Icarus, 181, 178

Cloutier, R., Astudillo-Defru, N., Doyon, R., et al. 2017, A\&A, 608, A35

Collins, K. A., Kielkopf, J. F., Stassun, K. G., \& Hessman, F. V. 2017, AJ, 153, 77

Cortés Contreras, M. 2016, PhD Thesis, Universidad Complutense de Madrid, Madrid, Spain

Cortés-Contreras, M., Béjar, V. J. S., Caballero, J. A., et al. 2017, A\&A, 597, A47

Crane, J. D., Shectman, S. A., Butler, R. P., et al. 2010, in Ground-based and Airborne Instrumentation for Astronomy III, Proc. SPIE, 7735, 773553

Cumming, A., Butler, R. P., Marcy, G. W., et al. 2008, PASP, 120, 531

Cuzzi, J. N., \& Zahnle, K. J. 2004, ApJ, 614, 490

Dai, F., Masuda, K., \& Winn, J. N. 2018, ApJ, 864, L38

Díez Alonso, E., Caballero, J. A., Montes, D., et al. 2019, A\&A, 621, A126

Dittmann, J. A., Irwin, J. M., Charbonneau, D., et al. 2017, Nature, 544, 333 
Drake, A. J., Graham, M. J., Djorgovski, S. G., et al. 2014, ApJS, 213, 9

Endl, M., Kürster, M., \& Els, S. 2000, A\&A, 362, 585

Espinoza, N. 2018, Res. Notes AAS, 2, 209

Espinoza, N., \& Jordán, A. 2015, MNRAS, 450, 1879

Espinoza, N., Kossakowski, D., \& Brahm, R. 2018, MNRAS, submitted [arXiv: 1812.08549]

Espinoza, N., Brahm, R., Henning, T., et al. 2019, MNRAS, submitted [arXiv:1903.07694]

Feroz, F., Hobson, M. P., \& Bridges, M. 2009, MNRAS, 398, 1601

Foreman-Mackey, D., Agol, E., Ambikasaran, S., \& Angus, R. 2017, celerite: Scalable 1D Gaussian Processes in $\mathrm{C}++$, Python, and Julia

France, K., Loyd, R. P., Youngblood, A., et al. 2016, ApJ, 820, 89

Fulton, B. J., Petigura, E. A., Blunt, S., \& Sinukoff, E. 2018, PASP, 130, 044504

Fulton, B. J., Petigura, E. A., Howard, A. W., et al. 2017, AJ, 154, 109

Gaia Collaboration (Brown, A. G. A., et al.) 2018, A\&A, 616, A1

Gandolfi, D., Barragán, O., Hatzes, A. P., et al. 2017, AJ, 154, 123

Gilliland, R. L., Marcy, G. W., Rowe, J. F., et al. 2013, ApJ, 766, 40

Gillon, M., Jehin, E., Lederer, S. M., et al. 2016, Nature, 533, 221

Gillon, M., Triaud, A. H. M. J., Demory, B.-O., et al. 2017, Nature, 542, 456

Gliese, W. 1957, Astronomisches Rechen-Institut Heidelberg Mitteilungen Serie A, 8,1

Greene, T. P., Line, M. R., Montero, C., et al. 2016, ApJ, 817, 17

Günther, M. N., Pozuelos, F. J., Dittmann, J. A., et al. 2019, ArXiv e-prints [arXiv: 1903.06107]

Harpsøe, K. B. W., Hardis, S., Hinse, T. C., et al. 2013, A\&A, 549, A10

Hawley, S. L., Gizis, J. E., \& Reid, I. N. 1996, AJ, 112, 2799

Hayano, Y., Takami, H., Oya, S., et al. 2010, in Adaptive Optics Systems II, Proc. SPIE, 7736, 77360N

Hébrard, E., Dobrijevic, M., Loison, J.-C., Bergeat, A., \& Hickson, K. 2012, A\&A, 541, A21

Iyer, A. R., Swain, M. R., Zellem, R. T., et al. 2016, ApJ, 823, 109

Jenkins, J. M., Twicken, J. D., McCauliff, S., et al. 2016, in Software and Cyberinfrastructure for Astronomy IV, Proc. SPIE, 9913, 99133E

Jensen, E. 2013, Tapir: A web interface for transit/eclipse observability, Astrophysics Source Code Library [record ascl: 1306.007]

Jódar, E., Pérez-Garrido, A., Díaz-Sánchez, A., et al. 2013, MNRAS, 429, 859

Johansen, A., Oishi, J. S., Mac Low, M.-M., et al. 2007, Nature, 448, 1022

Kaltenegger, L., \& Sasselov, D. 2010, ApJ, 708, 1162

Kasting, J. F., Whitmire, D. P., \& Reynolds, R. T. 1993, Icarus, 101, 108

Kawashima, Y., \& Rugheimer, S. 2019, AJ, 157, 213

Kempton, E. M.-R., Bean, J. L., Louie, D. R., et al. 2018, PASP, 130, 114401

Kipping, D. M. 2013, MNRAS, 435, 2152

Klahr, H., \& Bodenheimer, P. 2006, ApJ, 639, 432

Kochanek, C. S., Shappee, B. J., Stanek, K. Z., et al. 2017, PASP, 129, 104502

Kopparapu, R. K., Ramirez, R. M., SchottelKotte, J., et al. 2014, ApJ, 787, L29

Kostov, V. B., Schlieder, J. E., Barclay, T., et al. 2019, AJ, 158, 32

Kotani, T., Tamura, M., Nishikawa, J., et al. 2018, in Ground-based and Airborne Instrumentation for Astronomy VII, SPIE Conf. Ser., 10702, 107021

Kreidberg, L. 2015, PASP, 127, 1161

Kreidberg, L., Bean, J. L., Désert, J.-M., et al. 2014, Nature, 505, 69

Kürster, M., Endl, M., Rouesnel, F., et al. 2003, A\&A, 403, 1077

Labadie, L., Rebolo, R., Femenía, B., et al. 2010, in Ground-based and Airborne Instrumentation for Astronomy III, Proc. SPIE, 7735, 77350X

Lambrechts, M., Johansen, A., \& Morbidelli, A. 2014, A\&A, 572, A35

Lendl, M., Ehrenreich, D., Turner, O. D., et al. 2017, A\&A, 603, L5

Lenz, C. T., Klahr, H., \& Birnstiel, T. 2019, ApJ, 874, 36

Levison, H. F., Kretke, K. A., \& Duncan, M. J. 2015, Nature, 524, 322

Li, J., Tenenbaum, P., Twicken, J. D., et al. 2019, PASP, 131, 024506

Louie, D. R., Deming, D., Albert, L., et al. 2018, PASP, 130, 044401

Lovis, C., \& Pepe, F. 2007, A\&A, 468, 1115

Luque, R., Nowak, G., Pallé, E., et al. 2018, A\&A, 620, A171

Luyten, W. J. 1942, Publications of the Astronomical Observatory University of Minnesota (Minnesota, US: University of Minnesota Press), 2, 242

Mandel, K., \& Agol, E. 2002, ApJ, 580, L171

Marcy, G. W., Isaacson, H., Howard, A. W., et al. 2014, ApJS, 210, 20

Maxted, P. F. L., Anderson, D. R., Collier Cameron, A., et al. 2011, PASP, 123, 547

Mayor, M., Pepe, F., Queloz, D., et al. 2003, The Messenger, 114, 20

Ment, K., Dittmann, J. A., Astudillo-Defru, N., et al. 2019, AJ, 157, 32

Meschiari, S., Wolf, A. S., Rivera, E., et al. 2009, PASP, 121, 1016

Molaverdikhani, K., Henning, T., \& Mollière, P. 2019, ApJ, 873, 32

Mollière, P., Wardenier, J. P., van Boekel, R., et al. 2019, A\&A, 627, A67

Montes, D., López-Santiago, J., Gálvez, M. C., et al. 2001, MNRAS, 328, 45

Mordasini, C., Alibert, Y., Klahr, H., \& Henning, T. 2012, A\&A, 547, A111

Motalebi, F., Udry, S., Gillon, M., et al. 2015, A\&A, 584, A72

Moutou, C., Hébrard, E. M., Morin, J., et al. 2017, MNRAS, 472, 4563

Ofir, A. 2014, A\&A, 561, A138
Ofir, A., Dreizler, S., Zechmeister, M., \& Husser, T.-O. 2014, A\&A, 561, A103 Ofir, A., Xie, J.-W., Jiang, C.-F., Sari, R., \& Aharonson, O. 2018, ApJS, 234

Ormel, C. W., \& Klahr, H. H. 2010, A\&A, 520, A43

Ormel, C. W., Liu, B., \& Schoonenberg, D. 2017, A\&A, 604, A

Oscoz, A., Rebolo, R., López, R., et al. 2008, in Ground-based and Airborne Instrumentation for Astronomy II, Proc. SPIE, 7014, 701447

Otor, O. J., Montet, B. T., Johnson, J. A., et al. 2016, AJ, 152, 165

Pallé, E., Zapatero Osorio, M. R., Barrena, R., Montañés-Rodríguez, P., \& Martín, E. L. 2009, Nature, 459, 814

Parviainen, H. 2015, MNRAS, 450, 3233

Pojmanski, G. 2002, Acta Astron., 52, 397

Pollacco, D. L., Skillen, I., Collier Cameron, A., et al. 2006, PASP, 118, 1407 Quinn, S. N., White, T. R., Latham, D. W., et al. 2015, ApJ, 803, 49

Quirrenbach, A., Amado, P. J., Caballero, J. A., et al. 2014, in Ground-based and Airborne Instrumentation for Astronomy V, Proc. SPIE, 9147, 91471F

Quirrenbach, A., Amado, P. J., Ribas, I., et al. 2018, in Ground-based and Airborne Instrumentation for Astronomy VII, SPIE Conf.Ser., 10702 , 107020W

Reiners, A., Zechmeister, M., Caballero, J. A., et al. 2018, A\&A, 612, A49

Ribas, I., Tuomi, M., Reiners, A., et al. 2018, Nature, 563, 365

Ricker, G. R., Winn, J. N., Vanderspek, R., et al. 2015, J. Astron. Telesc. Instrum. Syst., 1, 014003

Rowe, J. F., Bryson, S. T., Marcy, G. W., et al. 2014, ApJ, 784, 45

Sarkis, P., Henning, T., Kürster, M., et al. 2018, AJ, 155, 257

Schöfer, P., Jeffers, S. V., Reiners, A., et al. 2019, A\&A, 623, A44

Schoonenberg, D., \& Ormel, C. W. 2017, A\&A, 602, A21

Schweitzer, A., Passegger, V. M., Cifuentes, C., et al. 2019, A\&A, 625, A68

Sinukoff, E., Howard, A. W., Petigura, E. A., et al. 2016, ApJ, 827, 78

Skrutskie, M. F., Cutri, R. M., Stiening, R., et al. 2006, AJ, 131, 1163

Smith, J. C., Stumpe, M. C., Van Cleve, J. E., et al. 2012, PASP, 124, 1000

Southworth, J. 2011, MNRAS, 417, 2166

Speagle, J. S. 2019, MNRAS, submitted [arXiv:1904.02180]

Stassun, K. G., Oelkers, R. J., Pepper, J., et al. 2018, AJ, 156, 102

Stelzer, B., Marino, A., Micela, G., López-Santiago, J., \& Liefke, C. 2013, MNRAS, 431, 2063

Stevenson, D. J., \& Lunine, J. I. 1988, Icarus, 75, 146

Stumpe, M. C., Smith, J. C., Van Cleve, J. E., et al. 2012, PASP, 124, 985

Stumpe, M. C., Smith, J. C., Catanzarite, J. H., et al. 2014, PASP, 126, 100

Suárez Mascare no, A., Rebolo, R., González Hernández, J. I., \& Esposito, M. 2015, MNRAS, 452, 2745

Sullivan, P. W., Winn, J. N., Berta-Thompson, Z. K., et al. 2015, ApJ, 809, 77

Tal-Or, L., Trifonov, T., Zucker, S., Mazeh, T., \& Zechmeister, M. 2019, MNRAS, 484, L8

ter Braak, C. J. F., \& Vrugt, J. A. 2008, Stat. Comput., 18, 435

Trifonov, T., Kürster, M., Zechmeister, M., et al. 2018, A\&A, 609, A117

Trotta, R. 2008, Contemp. Phys., 49, 71

Tuomi, M., \& Anglada-Escudé, G. 2013, A\&A, 556, A111

Twicken, J. D., Catanzarite, J. H., Clarke, B. D., et al. 2018, PASP, 130, 064502

Udry, S., Bonfils, X., Delfosse, X., et al. 2007, A\&A, 469, L43

Van Eylen, V., Agentoft, C., Lundkvist, M. S., et al. 2018, MNRAS, 479, 4786

Vanderspek, R., Huang, C. X., Vanderburg, A., et al. 2019, ApJ, 871, L24

Venot, O., Hébrard, E., Agúndez, M., et al. 2012, A\&A, 546, A43

Vogt, S. S., Allen, S. L., Bigelow, B. C., et al. 1994, in Instrumentation in Astronomy VIII, eds. D. L. Crawford \& E. R. Craine, Proc. SPIE, 2198, 362

Vogt, S. S., Marcy, G. W., Butler, R. P., \& Apps, K. 2000, ApJ, 536, 902

Wakeford, H. R., Sing, D. K., Kataria, T., et al. 2017, Science, 356, 628

Woźniak, P. R., Vestrand, W. T., Akerlof, C. W., et al. 2004, AJ, 127, 2436

Zacharias, N., Finch, C. T., Girard, T. M., et al. 2013, AJ, 145, 44

Zechmeister, M., \& Kürster, M. 2009, A\&A, 496, 577

Zechmeister, M., Kürster, M., \& Endl, M. 2009, A\&A, 505, 859

Zechmeister, M., Reiners, A., Amado, P. J., et al. 2018, A\&A, 609, A12

Zeng, L., Sasselov, D. D., \& Jacobsen, S. B. 2016, ApJ, 819, 127

1 Instituto de Astrofísica de Canarias (IAC), 38205 La Laguna, Tenerife, Spain e-mail: rluque@iac.es

2 Departamento de Astrofísica, Universidad de La Laguna (ULL), 38206 La Laguna, Tenerife, Spain

3 Max-Planck-Institut für Astronomie, Königstuhl 17, 69117 Heidelberg, Germany

4 Institut für Astrophysik, Georg-August-Universität, FriedrichHund-Platz 1, 37077 Göttingen, Germany

5 Landessternwarte, Zentrum für Astronomie der Universität Heidelberg, Königstuhl 12, 69117 Heidelberg, Germany 
${ }^{6}$ Kavli Institute for Astrophysics and Space Research, Massachusetts Institute of Technology, Cambridge, MA 02139, USA

7 School of Physics and Astronomy, Queen Mary University of London, 327 Mile End Road, London, E1 4NS, UK

${ }^{8}$ Instituto de Astrofísica de Andalucía (IAA-CSIC), Glorieta de la Astronomía s/n, 18008 Granada, Spain

${ }^{9}$ Centro de Astrobiología (CSIC-INTA), ESAC, Camino bajo del castillo s/n, 28692 Villanueva de la Cañada, Madrid, Spain

${ }^{10}$ Harvard-Smithsonian Center for Astrophysics, 60 Garden St, Cambridge, MA 02138, USA

11 Department of Physics and Astronomy, Vanderbilt University, Nashville, TN 37235, USA

12 Departamento de Física de la Tierra y Astrofísica \& IPARCOSUCM (Instituto de Física de Partículas y del Cosmos de la UCM), Facultad de Ciencias Físicas, Universidad Complutense de Madrid, 28040 Madrid, Spain

13 Department of Exploitation and Exploration of Mines, University of Oviedo, Oviedo, Spain

${ }^{14}$ Department of Terrestrial Magnetism, Carnegie Institution for Science, 5241 Broad Branch Road, NW, Washington, DC 20015, USA

15 Thüringer Landessternwarte Tautenburg, Sternwarte 5, 07778 Tautenburg, Germany

16 Astrophysics Group, Keele University, Staffordshire, ST5 5BG, UK

17 Carl Sagan Institute, Cornell University, Ithaca, NY 14853, USA

18 Astrobiology Center, 2-21-1 Osawa, Mitaka, Tokyo 181-8588, Japan

19 JST, PRESTO, 2-21-1 Osawa, Mitaka, Tokyo 181-8588, Japan

20 National Astronomical Observatory of Japan, 2-21-1 Osawa, Mitaka, Tokyo 181-8588, Japan

${ }^{21}$ Weizmann Institute of Science, 234 Herzl Street, Rehovot 761001, Israel

22 Department of Astrophysical Sciences, Princeton University, 4 Ivy Lane, Princeton, NJ 08544, USA
${ }^{23}$ Centro de Astrobiología (CSIC-INTA), Carretera de Ajalvir km 4, 28850 Torrejón de Ardoz, Madrid, Spain

24 Department of Astronomy and Astrophysics, University of California, Santa Cruz, CA 95064, USA

25 SETI Institute, Mountain View, CA 94043, USA

${ }^{26}$ NASA Ames Research Center, Moffett Field, CA 94035, USA

27 Key Laboratory of Planetary Sciences, Purple Mountain Observatory, Chinese Academy of Sciences, Nanjing 210008, PR China

28 Observatories of the Carnegie Institution for Science, 813 Santa Barbara Street, Pasadena, CA 91101, USA

29 Department of Astronomy, The University of California, Berkeley, CA 94720, USA

30 Institut de Ciències de l'Espai (ICE, CSIC), Campus UAB, C/Can Magrans s/n, 08193 Bellaterra, Spain

${ }^{31}$ Institut d'Estudis Espacials de Catalunya (IEEC), 08034 Barcelona, Spain

32 The Maury Lewin Astronomical Observatory, Glendora, California 91741, USA

33 Proto-Logic LLC, 1718 Euclid Street NW, Washington, DC 20009, USA

${ }^{34}$ Hamburger Sternwarte, Universität Hamburg, Gojenbergsweg 112, 21029 Hamburg, Germany

35 Centro Astronómico Hispano-Alemán (CSIC-MPG), Observatorio Astronómico de Calar Alto, Sierra de los Filabres-04550 Gérgal, Almería, Spain

36 School of Physical Sciences, The Open University, Milton Keynes MK7 6AA, UK

37 Department of Earth, Atmospheric and Planetary Sciences, Massachusetts Institute of Technology, Cambridge, MA 02139, USA

38 Department of Aeronautics and Astronautics, MIT, 77 Massachusetts Avenue, Cambridge, MA 02139, USA

39 Department of Astronomy, The University of Tokyo, 7-3-1 Hongo, Bunkyo-ku, Tokyo 113-0033, Japan 


\section{Appendix A: Joint fit priors}

Table A.1. Priors used for the joint fit model 3pl+GPexp presented in Sect. 5.2.3 using juliet.

\begin{tabular}{|c|c|c|c|}
\hline Parameter name & Prior & Units & Description \\
\hline \multicolumn{4}{|c|}{ Stellar parameters } \\
\hline$\rho_{\star}$ & $\mathcal{N}\left(13600,1700^{2}\right)$ & $\mathrm{kg} \mathrm{m}^{-3}$ & Stellar density \\
\hline \multicolumn{4}{|c|}{ Planet parameters } \\
\hline$P_{\mathrm{b}}$ & $\mathcal{N}\left(3.93079,0.001^{2}\right)$ & $\mathrm{d}$ & Period of planet $b$ \\
\hline$P_{\mathrm{c}}$ & $\mathcal{N}\left(9.1,0.1^{2}\right)$ & $\mathrm{d}$ & Period of planet c \\
\hline$P_{\mathrm{d}}$ & $\mathcal{N}\left(55.7,0.5^{2}\right)$ & d & Period of planet $\mathrm{d}$ \\
\hline$t_{0, b}-2450000$ & $\mathcal{N}\left(8517.99,0.1^{2}\right)$ & $d$ & Time of transit-center of planet $b$ \\
\hline$t_{0, c}-2450000$ & $\mathcal{U}(8312,8318)$ & d & Time of transit-center of planet c \\
\hline$t_{0, d}-2450000$ & $\mathcal{U}(8310,8340)$ & $\mathrm{d}$ & Time of transit-center of planet $d$ \\
\hline$r_{1, b}$ & $\mathcal{U}(0,1)$ & $\ldots$ & Parametrization for $p$ and $b$ \\
\hline$r_{2, b}$ & $\mathcal{U}(0,1)$ & $\ldots$ & Parametrization for $p$ and $b$ \\
\hline$K_{b}$ & $\mathcal{U}(0,10)$ & $\mathrm{m} \mathrm{s}^{-1}$ & RV semi-amplitude of planet $b$ \\
\hline$K_{c}$ & $\mathcal{U}(0,10)$ & $\mathrm{m} \mathrm{s}^{-1}$ & RV semi-amplitude of planet c \\
\hline$K_{d}$ & $\mathcal{U}(0,10)$ & $\mathrm{m} \mathrm{s}^{-1}$ & RV semi-amplitude of planet $\mathrm{d}$ \\
\hline $\mathcal{S}_{1, b, c, d}=\sqrt{e_{b, c, d}} \sin \omega_{b, c, d}$ & 0.0 (fixed) & $\ldots$ & Parametrization for $e$ and $\omega$ \\
\hline $\mathcal{S}_{2, b, c, d}=\sqrt{e_{b, c, d}} \cos \omega_{b, c, d}$ & 0.0 (fixed) & $\ldots$ & Parametrization for $e$ and $\omega$ \\
\hline \multicolumn{4}{|c|}{ Photometry parameters } \\
\hline$D_{\mathrm{TESS}}$ & 1.0 (fixed) & $\ldots$ & Dilution factor \\
\hline$M_{\mathrm{TESS}}$ & $\mathcal{N}\left(0,0.1^{2}\right)$ & ppm & Relative flux offset for TESS \\
\hline$\sigma_{\text {TESS }}$ & $\mathcal{U}(1,500)$ & ppm & Extra jitter term for TESS \\
\hline$q_{1, \mathrm{TESS}}$ & $\mathcal{U}(0,1)$ & $\ldots$ & Quadratic limb-darkening parametrization for TESS \\
\hline$q_{2, \mathrm{TESS}}$ & $\mathcal{U}(0,1)$ & $\ldots$ & Quadratic limb-darkening parametrization for TESS \\
\hline$M_{\mathrm{LCO}}$ & $\mathcal{N}\left(0,0.1^{2}\right)$ & ppm & Relative flux offset for LCO \\
\hline$\sigma_{\mathrm{LCO}}$ & $\mathcal{U}(1,2000)$ & ppm & Extra jitter term for $\mathrm{LCO}$ \\
\hline$q_{1, \mathrm{LCO}}$ & $\mathcal{U}(0,1)$ & $\ldots$ & Linear limb-darkening parametrization for $\mathrm{LCO}$ \\
\hline \multicolumn{4}{|c|}{$R V$ parameters } \\
\hline$\mu_{\text {HIRES }}$ & $\mathcal{U}(-10,10)$ & $\mathrm{m} \mathrm{s}^{-1}$ & Systemic velocity for HIRES \\
\hline$\sigma_{\text {HIRES }}$ & $\mathcal{U}(0,10)$ & $\mathrm{m} \mathrm{s}^{-1}$ & Extra jitter term for HIRES \\
\hline$\mu_{\mathrm{UVES}}$ & $\mathcal{U}(-10,10)$ & $\mathrm{m} \mathrm{s}^{-1}$ & Systemic velocity for UVES \\
\hline$\sigma_{\text {UVES }}$ & $\mathcal{U}(0,10)$ & $\mathrm{m} \mathrm{s}^{-1}$ & Extra jitter term for UVES \\
\hline$\mu_{\text {HARPS }}$ & $\mathcal{U}(-10,10)$ & $\mathrm{m} \mathrm{s}^{-1}$ & Systemic velocity for HARPS \\
\hline$\sigma_{\text {HARPS }}$ & $\mathcal{U}(0,10)$ & $\mathrm{m} \mathrm{s}^{-1}$ & Extra jitter term for HARPS \\
\hline$\mu_{\text {PFSpre }}$ & $\mathcal{U}(-10,10)$ & $\mathrm{m} \mathrm{s}^{-1}$ & Systemic velocity for PFSpre \\
\hline$\sigma_{\text {PFSpre }}$ & $\mathcal{U}(0,10)$ & $\mathrm{m} \mathrm{s}^{-1}$ & Extra jitter term for PFSpre \\
\hline$\mu_{\mathrm{PFSpost}}$ & $\mathcal{U}(-10,10)$ & $\mathrm{m} \mathrm{s}^{-1}$ & Systemic velocity for PFSpost \\
\hline$\sigma_{\text {PFSpost }}$ & $\mathcal{U}(0,10)$ & $\mathrm{m} \mathrm{s}^{-1}$ & Extra jitter term for PFSpost \\
\hline$\mu_{\text {CARMENES }}$ & $\mathcal{U}(-10,10)$ & $\mathrm{m} \mathrm{s}^{-1}$ & Systemic velocity for CARMENES \\
\hline$\sigma_{\text {CARMENES }}$ & $\mathcal{U}(0,10)$ & $\mathrm{m} \mathrm{s}^{-1}$ & Extra jitter term for CARMENES \\
\hline \multicolumn{4}{|c|}{ GP hyperparameters } \\
\hline & $\mathcal{J}\left(10^{-2}, 500\right)$ & ppm & Amplitude of GP component for TESS \\
\hline$T_{\mathrm{GP}, \mathrm{TESS}}$ & $\mathcal{J}\left(10^{-2}, 1000\right)$ & d & Length scale of GP component for TESS \\
\hline$\sigma_{\mathrm{GP}, \mathrm{RV}}$ & $\mathcal{J}(0.1,10)$ & $\mathrm{m} \mathrm{s}^{-1}$ & Amplitude of GP component for the RVs \\
\hline$T_{\mathrm{GP}, \mathrm{RV}}$ & $\mathcal{J}\left(10^{-4}, 10\right)$ & d & Length scale of GP component for the RVs \\
\hline
\end{tabular}

Notes. The prior labels of $\mathcal{N}, \mathcal{U}$, and $\mathcal{J}$ represent normal, uniform, and Jeffrey's distributions. The parametrization for $(p, b)$ using $\left(r_{1}, r_{2}\right)$ (Espinoza 2018) and the linear $\left(q_{1}\right)$ and quadratic $\left(q_{1}, q_{2}\right)$ limb-darkening parametrization (Kipping 2013) are both described in Sect. 5.2.1. 
Appendix B: RV time series of best joint fit
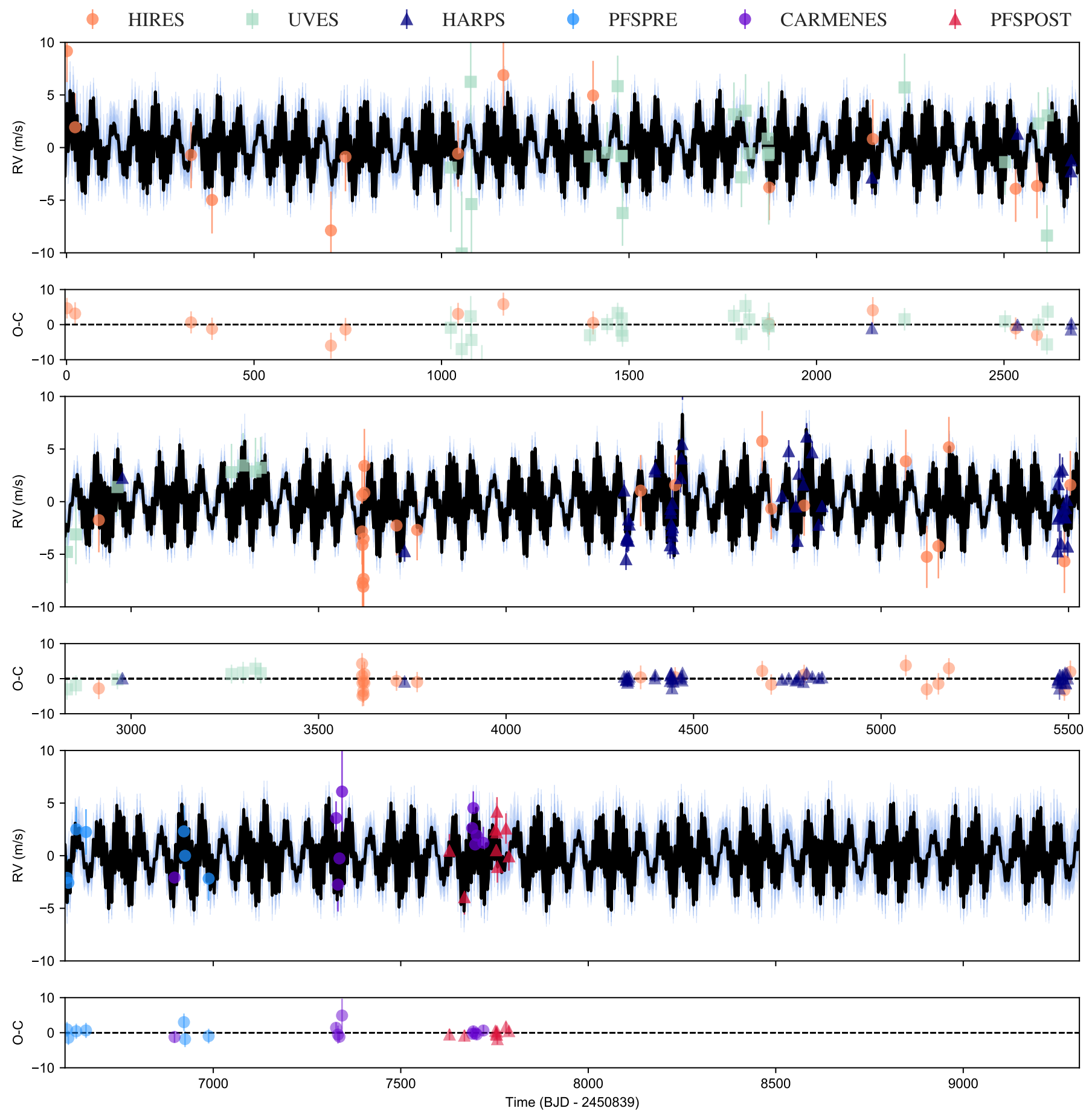

Fig. B.1. RV measurements as a function of time along with the residuals obtained from subtracting our median best joint fit model (black line) and the 68,95 , and $99 \%$ posterior bands (shown in blue). The color coding of the datapoints for each instrument is shown at the top. 